\title{
Hot coronal loop oscillations observed with SUMER: Examples and statistics ${ }^{\star}$
}

\author{
T. J. Wang ${ }^{1}$, S. K. Solanki ${ }^{1}$, W. Curdt ${ }^{1}$, D. E. Innes ${ }^{1}$, I. E. Dammasch ${ }^{1}$, and B. Kliem ${ }^{2}$ \\ 1 Max-Planck Institut für Aeronomie, 37191 Katlenburg-Lindau, Germany \\ 2 Astrophysikalisches Institut Potsdam, An der Sternwarte 16, 14482 Potsdam, Germany
}

Received 31 March 2003 / Accepted 27 May 2003

\begin{abstract}
We give an extensive overview of Doppler shift oscillations in hot active region loops obtained with SUMER. The oscillations have been detected in loops sampled 50-100 arcsec off the limb of the Sun in ultraviolet lines, mainly Fe XIX and Fe XXI, with formation temperature greater than 6 MK. The spectra were recorded along a 300 arcsec slit placed at a fixed position in the corona above the active regions. Oscillations are usually seen along an extended section of the slit and often appear to be from several different portions of the loops (or from different loops). Different portions are sometimes in phase, sometimes out of phase and sometimes show phase shifts along the slit. We measure physical parameters of 54 Doppler shift oscillations in 27 flare-like events and give geometric parameters of the associated hot loops when soft X-ray (SXR) images are available. The oscillations have periods in the range 7-31 min, with decay times 5.7-36.8 min, and show an initial large Doppler shift pulse with peak velocities up to $200 \mathrm{~km} \mathrm{~s}^{-1}$. The oscillation periods are on average a factor of three longer than the TRACE transverse loop oscillations. The damping times and velocity amplitude are roughly the same, but the derived displacement amplitude is four or five times larger than the transverse oscillation amplitude measured in TRACE images. Unlike TRACE oscillations, only a small fraction of them are triggered by large flares, and they often recur 2-3 times within a couple of hours. All recurring events show initial shifts of the same sign. These data provide the following evidence to support the conclusion that these oscillations are slow magnetoacoustic standing waves in hot loops: (1) the phase speeds derived from observed periods and loop lengths roughly agree with the sound speed; (2) the intensity fluctuation lags the Doppler shifts by $1 / 4$ period; (3) The scaling of the dissipation time of slow waves with period agrees with the observed scaling for 49 cases. They seem to be triggered by micro- or subflares near a footpoint, as revealed in one example with SXR image observations. However other mechanisms cannot as yet be ruled out. Some oscillations showed phase propagation along the slit in one or both directions with apparent speeds in the range of 8-102 $\mathrm{km} \mathrm{s}^{-1}$, together with distinctly different intensity and line width distributions along the slit. These features can be explained by the excitation of the oscillation at a footpoint of an inhomogeneous coronal loop, e.g. a loop with fine structure.
\end{abstract}

Key words. Sun: activity - Sun: corona - Sun: oscillations - Sun: UV radiation - Sun: X-rays, gamma rays

\section{Introduction}

MHD waves are believed to play an important role in the solar corona, e.g. as a possible source for heating of coronal loops. The waves can also be used as a tool to diagnose the physical parameters of the coronal plasma (e.g. Roberts et al. 1984; Nakariakov \& Ofman 2001). Various periodic and quasiperiodic oscillations in radio, visible, EUV, and soft X-ray (SXR) radiation have been observed for decades (e.g. reviews by Aschwanden 1987, 2002; Roberts 2000). Recently, temporally and spatially resolved transverse and longitudinal oscillations have been detected in coronal loops by the Solar and Heliospheric Observatory (SOHO) and the Transition Region and Coronal Explorer (TRACE). For example, signatures of

\footnotetext{
Send offprint requests to: T. J. Wang, e-mail: wangtj@linmpi.mpg.de

* Table 1 and Appendices A and B are only available in electronic form at http://www. edpsciences.org
}

propagating compressive waves were first observed in coronal loops by the EUV Imaging Telescope (EIT) (Berghmans \& Clette 1999), later confirmed by TRACE observations (De Moortel et al. 2000, 2002a,b,c; Robbrecht et al. 2001), and identified as propagating slow waves (Nakariakov et al. 2000; Tsiklauri \& Nakariakov 2001). Kink mode oscillations excited by flares in coronal loops were for the first time detected by TRACE in EUV radiation (Aschwanden et al. 1999b; Nakariakov et al. 1999). An extensive overview and analysis of transverse loop oscillations was presented by Schrijver et al. (2002) and a detailed discussion of the parameters obtained from these observations can be found in Aschwanden et al. (2002). The observed rapid damping of these transverse oscillations has been explained by anomalously high viscosity or resistivity due to resonant absorption (Nakariakov et al. 1999), or phase mixing (Ofman 2002; Ofman \& Aschwanden 2002). Schrijver \& Brown (2000) proposed an alternative mechanism: loop oscillations are caused by rocking motions of the 
photospheric plasma associated with flares if the loop lies near magnetic nullpoints or separators. They attribute the rapid decay of the oscillation to photospheric properties rather than the coronal resonant response. This model can explain why only a small subset of the loops visible in TRACE images are involved in oscillations.

Strongly damped Doppler shift oscillations in hot flare lines recorded by the Solar Ultraviolet Measurements of Emitted Radiation (SUMER) spectrometer on SOHO were recently discovered by Kliem et al. (2002) and identified as oscillations of hot coronal loops (Wang et al. 2002a,b,c). On the basis of the initial observations alone it was not directly possible to distinguish between transverse and longitudinal oscillations. Based on a one-dimensional MHD model, Ofman \& Wang (2002) found that due to the high temperature of the loops, the large thermal conduction can lead to rapid damping of slow-mode magnetoacoustic waves on a timescale comparable to those observed, and proposed that the Doppler oscillations are indeed due to the slow-mode waves. Strong observational evidence for slow-mode standing waves in high temperature postflare loops was recently provided by Wang et al. (2003). These investigations are limited in that they only consider a few individual events, so that an overview is as yet not available in the refereed literature.

In this paper we present an extensive overview of physical properties of hot loop oscillations in 27 events (each of which may harbour multiple oscillations), which are found by inspecting nearly all SUMER observations of limb active regions during the past three years. In Sect. 2, the observations and data processing are described. In Sect. 3, we present a detailed analysis of 6 selected examples. In Sect. 4, we provide an overview of the obtained parameters and compare with results of the TRACE transverse loop oscillations. In Sect. 5, we discuss the mode, damping, and trigger of the oscillations, and present conclusions in Sect. 6. Finally, Appendix A describes a method to derive geometrical parameters for the limb loops, and Appendix B gives a graphical overview of analyzed events not described in Sect. 3.

\section{Observations and data processing}

In order to study the variability and dynamics of active region loops, a number of spectral observations were recorded by SUMER (Wilhelm et al. 1995) in recent SOHO campaigns. In all cases, the SUMER spectrometer slit was placed at a fixed position in the corona about $100^{\prime \prime}$ above an active region at the limb. The observations of AR 8758 on 6 November 1999 are described in detail by Kliem et al. (2002). They covered 8 lines in the range $1320-1360 \AA$ with the $300^{\prime \prime} \times 1^{\prime \prime}$ slit and used an exposure time of $120 \mathrm{~s}$. The observations of AR 9371 on 9 March 2001 and AR 9176 on 29 September 2000 were made in the spectral range $1098-1138 \AA$, with a 162 s exposure time and the $300^{\prime \prime} \times 4^{\prime \prime}$ slit. This spectral window contains lines formed in the temperature range $0.01-10 \mathrm{MK}$, e.g. the relatively cool transition region line, a blend of S III/Si III $\lambda 1113$ $(0.03-0.06 \mathrm{MK})$, the coronal lines $\mathrm{Ca} \mathrm{X} \lambda 557 \times 2(0.7 \mathrm{MK})$ and Ca XIII $\lambda 1134$ (2 MK), as well as the flare lines Fe XIX $\lambda 1118$ $(6.3 \mathrm{MK})$ and $\mathrm{Fe} \mathrm{XX} \lambda 567 \times 2(8 \mathrm{MK})$. Standard procedures
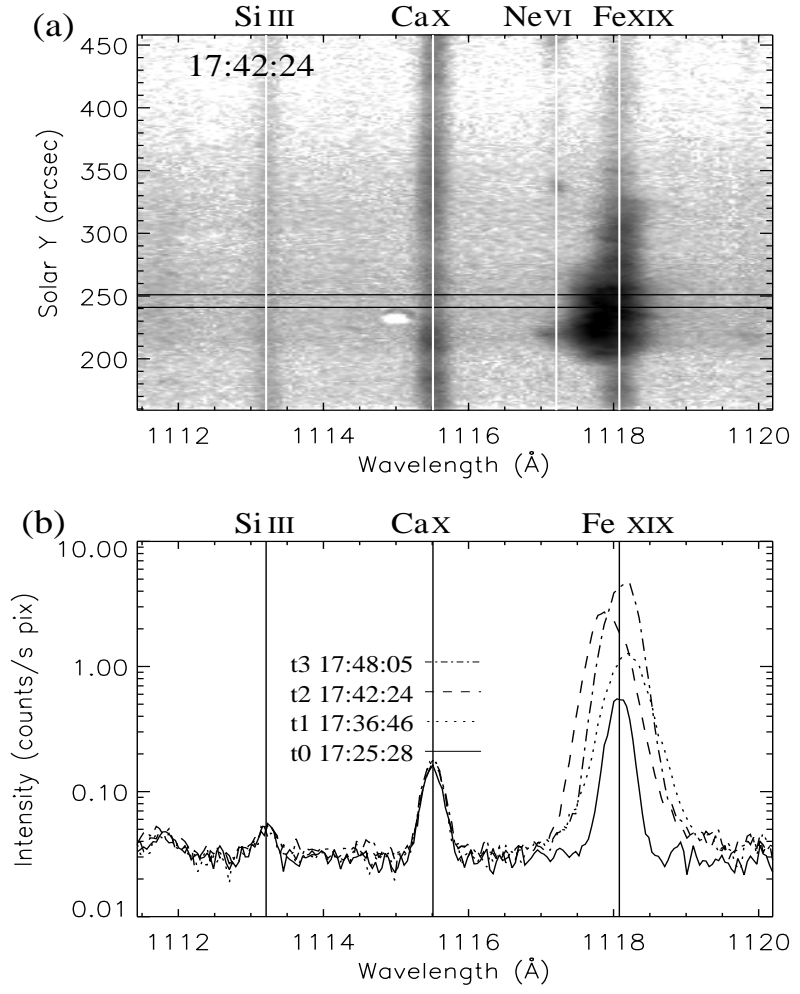

Fig. 1. a) The SUMER spectrum above an active region at the limb, taken at 17:42:24 UT on 9 March 2001 with an exposure time of $162 \mathrm{~s}$. The wavelength positions of 4 selected spectral lines are indicated by the vertical white lines. b) Line spectra are obtained by averaging the emission along the section of the slit marked by a strip in a). t0 is the time just before the oscillation event; $\mathrm{t} 1, \mathrm{t} 2$ and $\mathrm{t} 3$ the times of the first three Doppler shift peaks (Figs. 2a and 2b), respectively. Figure 1a corresponds to $\mathrm{t} 2$.

for decompression and corrections of flat-field, detector distortions, deadtime, and gain effects were applied to the raw data. Figure 1 illustrates the evolution of 4 spectral lines (Si III, Ca X, Ne VI [0.3 MK], and Fe XIX) in a selected window. The Doppler shift oscillations were detected only in the hot flare line Fe XIX without any signature in the other lines (Fig. 1b), but perhaps the Ne VI line is too weak and Si III is marginal in strength to show clearly recognizable oscillations. The oscillation periods measured from these observations are 11.7-31.1 min (see Sect. 4), but shorter periods could not be excluded for these observations because of the cadence of $>2$ min leading to a 4-point resolution limit of $\sim 10 \mathrm{~min}$ (Wang et al. 2002a,b).

Observations with a high cadence of $50 \mathrm{~s}$ were made in April-May 2002. This cadence allows us to detect oscillations with a period as short as $3 \mathrm{~min}$, but at the cost of not being able to transmit the whole $40 \AA$ wavelength band. The spectra were usually recorded in three lines Si III, Ca X and Fe XIX, or in C II $\lambda 1335$ (0.016 MK), Fe XII $\lambda 1349$ (1.6 MK) and Fe XXI $\lambda 1354$ (8.9 MK). In two cases other combinations of spectral lines were recorded, with a different cadence. The events on 8 May 2002 were observed in four lines Fe XIX, Fe XVII $\lambda 1153$ (2.8 MK), CaXIII and CaX with a 64 s cadence. The event on 15 May 2002 was observed in lines CII, 

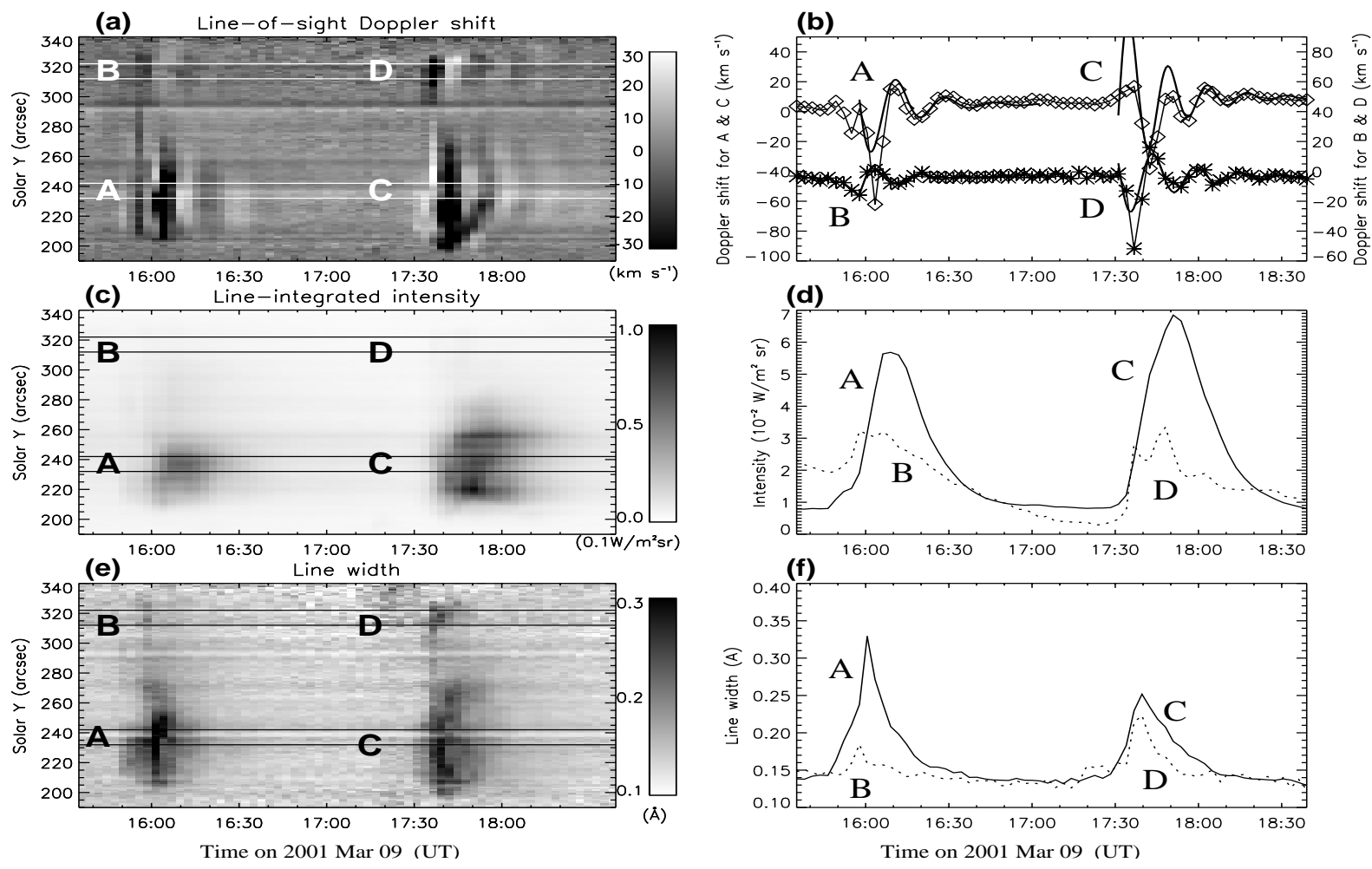

Fig. 2. Doppler oscillation events (Nos. 5A, 5B, 6C, 6D in Table 1) in the Fe XIX line on 9 March 2001. a) Doppler shift time series. The redshift is represented with the bright color, and the blueshift with the dark color. b) Average time profiles of Doppler shifts along cuts $A C$ and $B D$. The thick solid curves are the best fit functions described by Eq. (1). c) Line-integrated intensity time series. d) Average time profiles of line-integrated intensities along cuts $A C$ and $B D$. For a clear comparison, the intensity profile for $B D$ has been stretched by a factor of 10 . e) Line width (measured Gaussian width) time series. f) Average time profiles of line width along cuts $A C$ and $B D$.

Fe XII and Fe XXI (as well as its two adjacent windows) with a 75 s cadence.

After processing the raw data following standard procedures, a single Gaussian was fit to each line profile to obtain a Doppler shift time series at each spatial pixel (e.g. Fig. 2a). A large number of impulsive brightenings in the hot flare lines Fe XIX or Fe XXI are revealed associated with alternating red and blue Doppler shifts. However, many of them were not analyzed further since the Doppler signal was either: (a) nonperiodic, (b) visible only over one period, or (c) was very weak. In this study, we identify the Doppler shift oscillations only for those cases of relatively regular period, which lasted for more than 1.5 periods and exhibited a maximum Doppler shift $>10 \mathrm{~km} \mathrm{~s}^{-1}$. Following this rule, we found the $27 \mathrm{os}-$ cillation events listed in Table 1 . The meaning of the symbols heading the various columns is described in a footnote to the table. In some events, several oscillation components were identified along the slit due to differences in period or phase. For each oscillation component, we averaged over a width of 11 pixels $\left(\sim 1^{\prime \prime}\right.$ pixel $\left.^{-1}\right)$ for the data sets observed during 1999-2001 and 6 pixels for the data sets observed in 2002 to reduce noise when plotting and analyzing the average time profile. The function

$V(t)=V_{0}+V_{\mathrm{D}} \sin (\omega t+\phi) \mathrm{e}^{-\lambda t}$,

was then fit to the oscillation, where $V_{0}$ is the background Doppler shift, $V_{\mathrm{D}}$ is the Doppler shift amplitude and $\omega, \phi$, and $\lambda$ are the frequency, phase, and decay rate of the oscillations. We define $V_{\mathrm{m}}$ as the maximum Doppler shift amplitude measured from the data, relative to $V_{0}$. We derive the maximum displacement amplitude by $A=V_{\mathrm{m}} /\left(\omega^{2}+\lambda^{2}\right)^{1 / 2}$. The obtained parameters of the time series are listed in Table 1.

For some events the soft X-ray telescope (SXT) (Tsuneta et al. 1991) on Yohkoh provided coordinated observations, allowing us to identify the oscillating loops and their geometric parameters. For the 9 March 2001 events, the SXT obtained full $\left(2\right.$. $^{\prime \prime}$ pixel $\left.^{-1}\right)$, half-, and quarter-resolution partial frame images only in the decay phase. For the 29 September 2000 events, the SXT obtained half-, and quarter-resolution full disc frame images with a 4.3 min cadence, covering the whole period of the events. We also identified the association of SUMER oscillation events with flares measured in the 1-8 $\AA$ channel of the GOES satellite, using EIT full disc images to examine if EUV flarings occurred in the limb active region SUMER observed. We list these flare-associated events in Table 2.

\section{Description and analysis of selected oscillation events}

In a total of 27 Doppler oscillation events, we describe 6 selected examples of various types of oscillations and analyze their physical properties, loop geometrical parameters and triggers in detail. The remaining cases are graphically shown in Appendix B in Figs. B.1-B.11. 
Table 2. Flare-associated Doppler oscillation events ${ }^{\mathrm{a}}$.

\begin{tabular}{lrcccccc}
\hline \hline \# Event & Date & AR & Flare & $t_{0}^{\text {GOES }}$ & $t_{\mathrm{m}}^{\text {GOES }}$ & $t_{0}^{\text {SUMER }}$ & $t_{\mathrm{m}}^{\text {SUMER }}$ \\
\hline 1 & 06-Nov.-99 & 8758 & C4.6 & $06: 28$ & $06: 36$ & $06: 30$ & $06: 40$ \\
4 & 29-Sep.-00 & 9176 & C4.5 & $13: 05$ & $13: 13$ & $13: 07$ & $13: 15$ \\
$17 / 18$ & 15/16-Apr.-02 & 9893 & M1.2 & $23: 05$ & $23: 24$ & $23: 08$ & $23: 56$ \\
25 & 08-May-02 & 9929 & C2.8 & $14: 39$ & $14: 45$ & $14: 44$ & $15: 10$ \\
26 & 08-May-02 & 9929 & C2.3 & $15: 46$ & $15: 52$ & $15: 44$ & $16: 12$ \\
27 & 15-May-02 & 9934 & M1.0 & $08: 00$ & $08: 13$ & $08: 08$ & $09: 07$ \\
\hline
\end{tabular}

a Listed are: (1) Doppler oscillation event number; (2) date; (3) NOAA active region number; (4) GOES flare class; (5) start time (UT) of GOES X-ray flux $\left(t_{0}^{\mathrm{GOES}}\right)$; (6) peak time (UT) of GOES X-ray flux $\left(t_{\mathrm{m}}^{\mathrm{GOES}}\right)$; (7) start time (UT) of Fe XIX or Fe XXI line-integrated intensity enhancement averaged along the SUMER slit; (8) peak time (UT) of the SUMER line-integrated intensity.

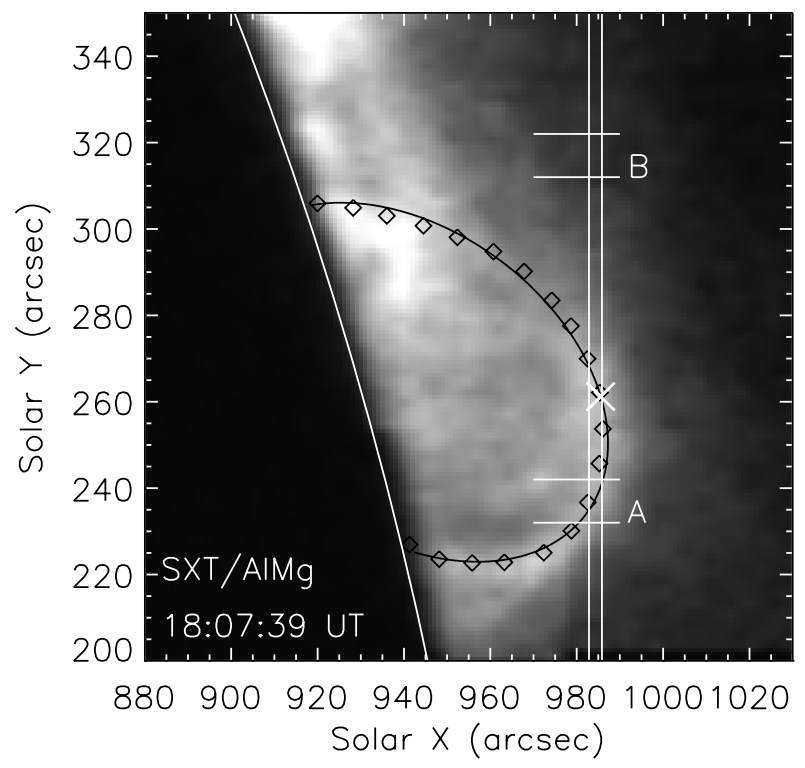

Fig. 3. The oscillating soft X-ray loop (outlined with diamonds) fitted with a circular model (dark curve) for the 9 March 2001 events. The apex position of the modelled loop is marked with a cross. The SUMER spectrometer slit position is indicated, and positions of two cuts (denoted A and B in Fig. 2) are marked.

\subsection{March 2001}

This example (Figs. 2 and 3) is probably the clearest case of loop oscillations, which was described in detail in Wang et al. (2002a). SUMER observed two hot plasma events occurring in AR 9371 at the west limb. No GOES flares were related to these two SUMER events. The oscillations have periods of 14-18 min, with decay times of 12-19 min. Figures 2e and $2 \mathrm{f}$ show variations of the measured Gaussian widths of the Fe XIX line. They show substantial Doppler broadenings in the rising phase of the flux, and the widths peak almost simultaneously with the shifts. For case $A$, the measured maximum Gaussian width, $v_{\sigma}=98 \mathrm{~km} \mathrm{~s}^{-1}$, so the Doppler width, $v_{\mathrm{D}}=\sqrt{2} v_{\sigma}=138 \mathrm{~km} \mathrm{~s}^{-1}$. We obtain the nonthermal velocity, $\xi=\sqrt{v_{\mathrm{D}}^{2}-v_{\mathrm{th}}^{2}}=132 \mathrm{~km} \mathrm{~s}^{-1}$, where $v_{\text {th }}=43 \mathrm{~km} \mathrm{~s}^{-1}$, the thermal velocity of the $\mathrm{Fe}^{+18}$ ion. The large turbulent velocity introduces a large uncertainty into the measurement of the initial Doppler shifts.
During the second event, when simultaneous SXT observations were available, a SXR loop was seen at the position of the loop oscillation (Fig. 3). Based on a circular model described in Appendix A, we derive the geometric parameters of the loop from measurements of the footpoint separation and the apex position of the loop. The obtained loop length, the inclination angle and the azimuth angle of the footpoint baseline are listed in Table 3. The loop length derived using the same method has been used to discuss wave modes in Wang et al. (2002a).

\subsection{November 1999}

In this case the oscillations are seen in the Fe XXI line, which are even better defined than in the March 9 events (Fig. 4). Because simultaneous SXT observations were available only at the very beginning of the event, we are unable to pinpoint the oscillating loop. This time the event was associated with a C4.6 flare that occurred in AR 8758 at the north-east limb. The enhancement in the line-integrated intensity in Fe XXI started and peaked later than the GOES X-ray flux by several minutes (Table 2). More details of the event and associated cool $\left(T \sim 10^{4} \mathrm{~K}\right.$ ) line emission are given by Kliem et al. (2002). A damped sine-function provides a good fit (Fig. 4b) and gives a period of $11.7 \mathrm{~min}$. The time series of the shifts and line widths are coherent along the slit.

\subsection{September 2000}

In this case, coordinated observations between SUMER and Yohkoh/SXT provide convincing evidence that the Doppler oscillation corresponds to oscillations of hot coronal loops. The SUMER time series revealed 4 hot plasma events, all in the Fe XIX line, without signatures in the lines formed below 2 MK. Figure 5 shows the two earlier events. The first at about 02:30 UT and the second about an hour later at 03:50 UT. Neither was detected by GOES. But in both events SXT images show a bright point near one footpoint $(F 1)$ of a large coronal loop (Fig. 6) which (the footpoint) reached maximum brightness around the time of the initial Doppler shift pulse seen by SUMER. The two later events, starting at about 10:10 UT and 13:10 UT (Fig. 7) were both associated with GOES C-class flares (Table 2). These two events were also associated with enhencements of the SXR bright point (Fig. 8), and with an 
Table 3. Geometrical parameters of oscillating loops derived from Yohkoh/SXT images ${ }^{\mathrm{a}}$.

\begin{tabular}{lcccccccc}
\hline \hline Time & $\begin{array}{c}l_{0}-l_{\odot} \\
(\mathrm{deg})\end{array}$ & $\begin{array}{c}b_{0}-b_{\odot} \\
(\mathrm{deg})\end{array}$ & $\begin{array}{c}\alpha^{\mathrm{b}} \\
(\mathrm{deg})\end{array}$ & $\begin{array}{c}\theta^{\mathrm{c}} \\
(\mathrm{deg})\end{array}$ & $\begin{array}{c}\psi \\
(\mathrm{deg})\end{array}$ & $\begin{array}{c}h_{0} \\
(\mathrm{Mm})\end{array}$ & $\begin{array}{l}a \text { and } b(\text { or } r) \\
(\mathrm{Mm})\end{array}$ & $\begin{array}{c}L \\
(\mathrm{Mm})\end{array}$ \\
\hline 02:24:49 29-Sep.-00 & -59.3 & -11.1 & 22.6 & 28.0 & 33.9 & 51 & $a=135$ and $b=66$ & 473 \\
10:13:23 29-Sep.-00 & -55.9 & -11.8 & 22.5 & 31.6 & 36.3 & 50 & $a=154$ and $b=63$ & 515 \\
18:07:39 09-Mar.-01 & 90.0 & 16.5 & 57.9 & 35.5 & 57.9 & 9 & $r=37$ & 135 \\
\hline
\end{tabular}

${ }^{\text {a }} t_{0}$ is the time of the modeled images. $l_{0}-l_{\odot}$ and $b_{0}-b_{\odot}$ are the heliographic longitude and latitude relative to Sun center for the midpoint of the loop footpoint baseline. We assume that $l_{\odot}=0$ and $b_{\odot}=0$ for the used heliographic coordinate system. $\alpha$ is the azimuth angle of the loop baseline to the east-west direction. $\theta$ is the inclination angle of the loop plane to the vertical. $\psi$ is the angle between the loop baseline and line-of-sight. $h_{0}$ is the height of the elliptical (or circular) loop center in the loop plane. $a$ and $b$ are the semi-major and semi-minor axis lengths of the elliptical loop, under the assumption that the semi-major axis is parallel to the solar surface; $r$ is the radius of the circular loop. $L$ is the loop length.

b The loop baseline is rotated counterclockwise by an angle $\alpha$ relative to the east-west direction in the 29-Sep.-00 cases; but the direction of rotation of the azimuth angle in the 09-Mar.-01 case is uncertain (see Appendix A).

${ }^{c}$ The loop plane is inclined northward by an angle $\theta$ relative to the vertical in the 29-Sep.-00 cases; but the direction of inclination in the 09-Mar.-01 case is uncertain (see Appendix A).

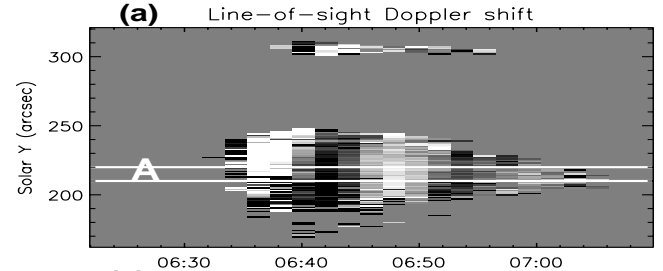

(c) $^{06: 30}$ Line-integrated intensity $06: 00$
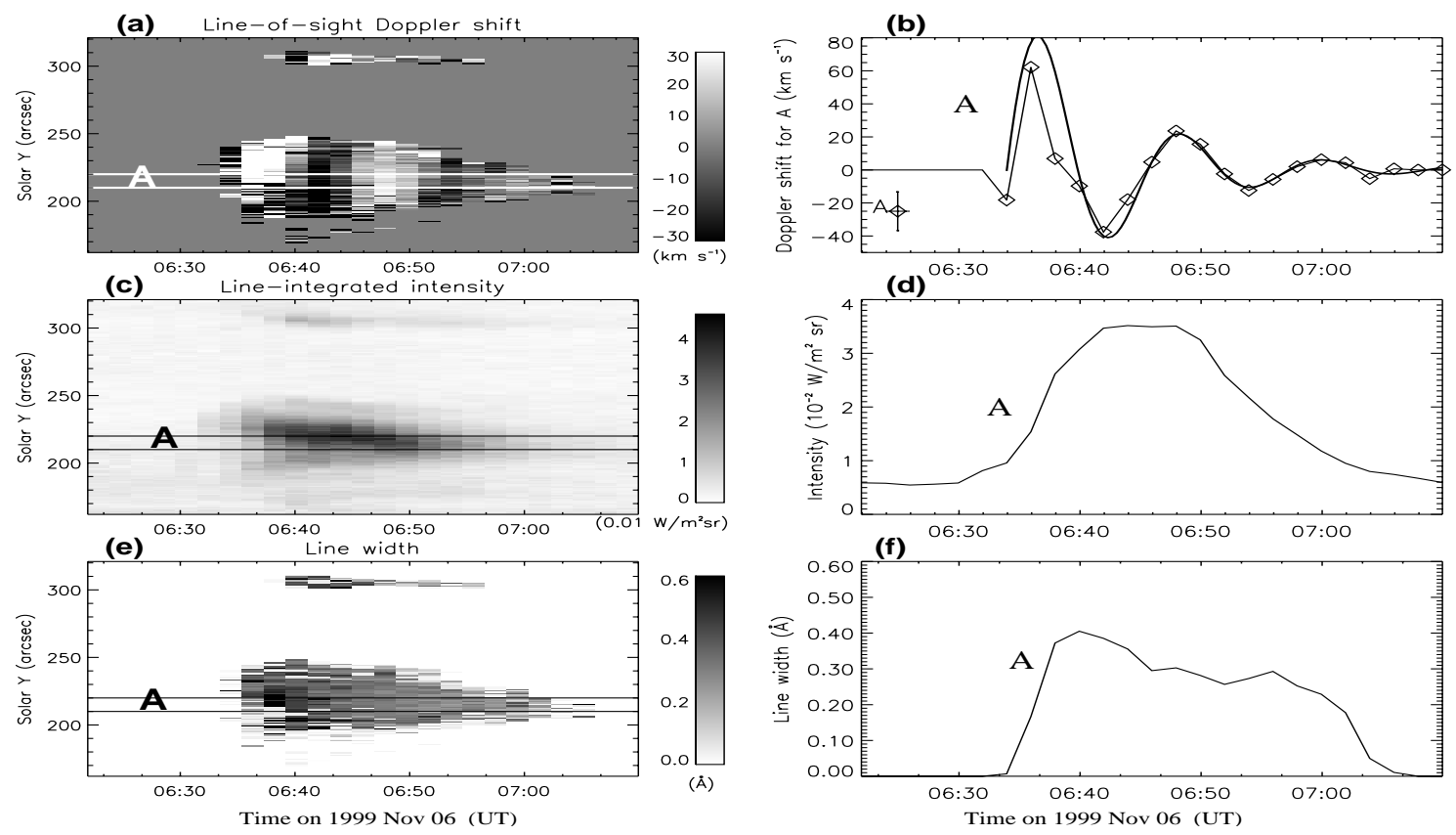

Fig. 4. Doppler oscillation event (No. 1A) recorded in the Fe XXI line on 6 November 1999. The figure is structured similarly to Fig. 2.

EUV brightening observed by EIT at the same site at the start of the oscillation. The EUV brightening showed a faint emission at its edge moving upward along the large coronal loop. We find that the earlier events are about a factor five fainter (Figs. 5d and 7d), and have well defined oscillations whereas the latter flare-associated events are much more complex with several non-periodic components. These oscillations have similar periods in the range $25-31 \mathrm{~min}$.

Figures 6 and 8 show that the SUMER slit was near the top of a large SXR loop, and the Doppler oscillations coincide with the regions where the slit crosses this loop. Figures $6 \mathrm{c}$ and $6 \mathrm{~d}$ show that the EUV loops differ in position from the SXR loop. During the fainter earlier events, the SXR loops brightened but there were no major changes in the loops' structure. Both flare events, however, were associated with X-ray plasma ejections (see Fig. 8) and this is probably why the Doppler shifts are confusing. Nevertheless, there are two regions $(A$ and $C)$ in the second flare event (around 13:20 UT) with in-phase oscillations (see Fig. 7a). These may correspond to the intersection of the slit with two legs of an oscillating loop (see Fig. 8c).

Assuming an elliptical shape, we determine the geometrical parameters of the SXR loop, by using a method similar to that employed by Aschwanden et al. (2002). This method optimizes 3 free parameters $\left(h_{0}, \theta, e\right)$ to obtain a good match with the observed loop (Figs. 6a and 8a), where $h_{0}$ is the height of the center of the ellipse above the solar surface, $\theta$ is the inclination angle of the loop plane to the vertical, and $e$ is the ellipticity. The obtained parameters are listed in Table 3. Comparing the parameters of the loop at 02:24 UT and at 10:13 UT, we find that the loop became longer, flatter and more inclined in 

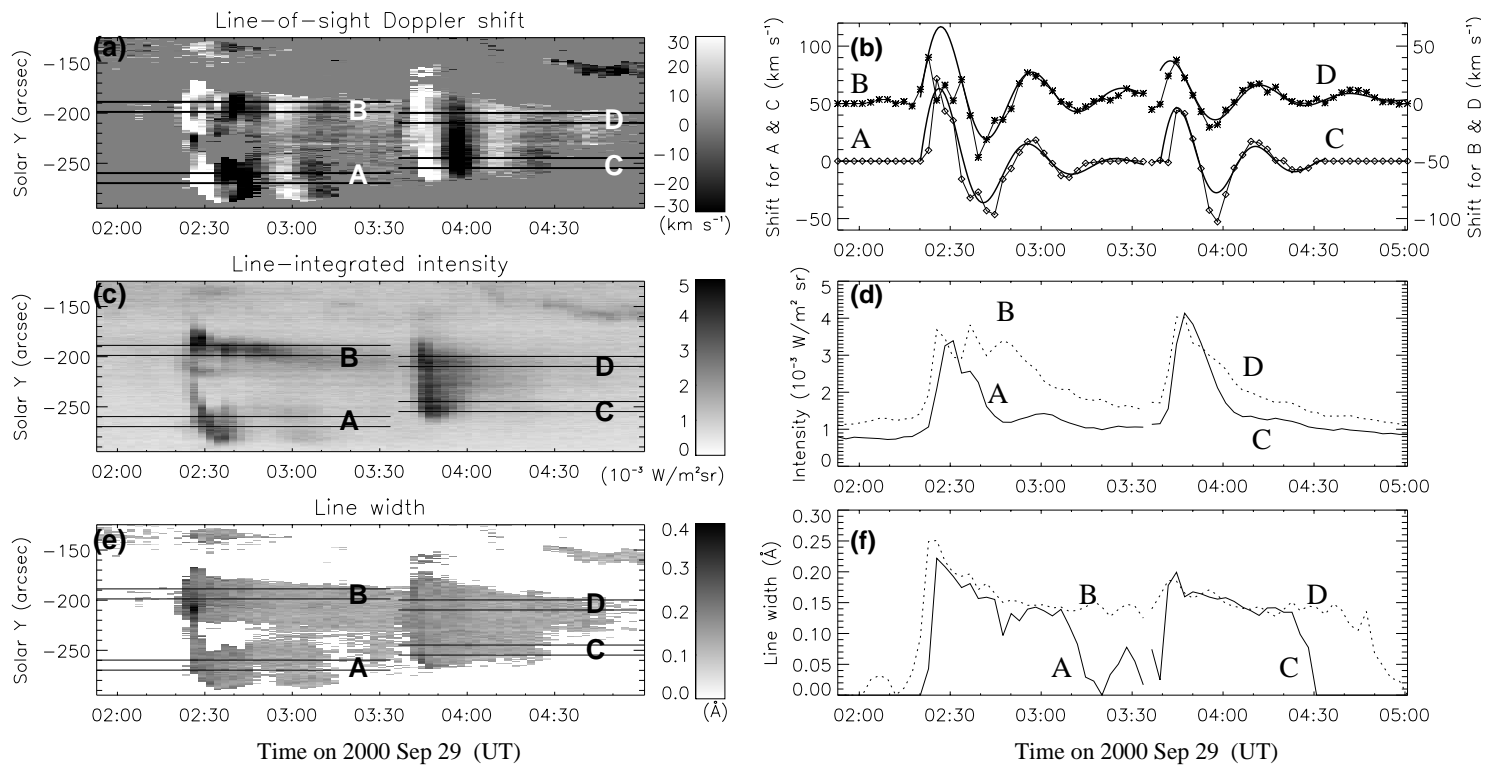

Fig. 5. Doppler oscillation events (Nos. 2A, 2B, 3C, 3D) in the Fe XIX line on 29 September 2000. See caption of Fig. 2 for more details.

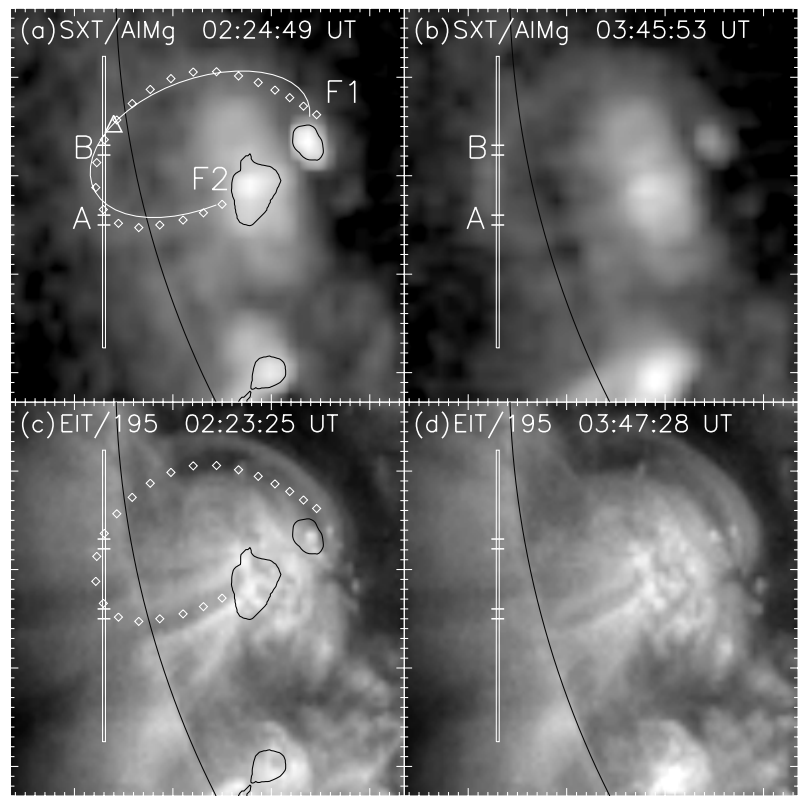

Fig. 6. a) The oscillating soft X-ray loop (outlined with diamonds) fitted with an elliptical model (white curve) for the 29 September 2000 events. The apex position of the modelled loop is marked with a triangle. The dark contours outline the X-ray brightenings. The SUMER spectrometer slit position is indicated, and positions of two cuts (denoted A and B in Fig. 5) are marked. b) The SXT image at 03:45:53 UT. c) The SOHO/EIT image overlaid with the SXR loop (diamonds) and SXR brightenings (dark contours) taken from a). d) The EIT image at 03:47:28 UT.

this interval, i.e. after the two earlier events. These features may suggest that the loop expanded and became heavier due to filling by hot plasma during these events.

From the 3-D geometry we derive tangential vectors to the loop (defined along the loop pointing toward the footpoint $F 2$ ) close to cuts $A$ and $B$ with the slit (see Fig. 6a). The angles to the line-of-sight are $138^{\circ}$ at $A$ and $150^{\circ}$ at $B$ for the image at 02:24 UT, respectively (Fig. 6a), and the angles are $118^{\circ}$ at $A$, $150^{\circ}$ at $B$ and $149^{\circ}$ at $C$ for the image at 10:13 UT (Fig. 8a). We notice that all 4 events began with strong red shifts and were associated with enhancements of a SXR bright point (or EIT brightening) near one footpoint of the large coronal loop. The calculated loop geometry implies that the initial strong red shifts are caused by an injected hot outflow or a flow-associated propagating disturbance from the footpoint brightening, which is probably due to magnetic reconnection between the large scale loop and a small scale flux system.

\subsection{April 2002}

This is an example of Doppler oscillations in the high cadence ( $\sim 50 \mathrm{~s})$ observations. Figure 9 shows that three oscillation events occurred within 2 hours, with periods in the range 13-18 $\mathrm{min}$ and decay times of 9-17 min. These events occurred at the east limb, with no associated GOES flares. In the second event, we find that the period and decay time vary along the slit. For example, cases $B$ and $D$ have a similar period of $\sim 17 \mathrm{~min}$, but distinctly different decay times of $9 \mathrm{~min}$ and $17 \mathrm{~min}$. The amplitudes also differ significantly. Thus, the maximum red- and blue-Doppler shifts reached by oscillation $B$ are 183 and $36 \mathrm{~km} \mathrm{~s}^{-1}$, while for case $D$, the corresponding shifts are 34 and $30 \mathrm{~km} \mathrm{~s}^{-1}$. Case $C$ has a period of $13 \mathrm{~min}$, i.e. distinctly shorter than cases $B$ and $D$, but its average intensity is about a factor 5 stronger than that of cases $B$ and $D$. Moreover, the high cadence observations reveal propagation (phase delay) of the Doppler shift oscillations along the slit from region $(C)$ of strong emission towards the fainter regions (e.g. $B$ and $D$ ). The propagation from $C$ to $B$ (cut 1 ) and that from $C$ to $D$ (cut 2) have durations of $150 \mathrm{~s}$ and $200 \mathrm{~s}$. The phase propagating speeds decrease with time. We measure the speeds from $C$ to $B$ as 96,49 , and $34 \mathrm{~km} \mathrm{~s}^{-1}$ from the slopes of cuts 1,3 and 5, while the speeds for $C$ to $D$ are 83,48 , and $13 \mathrm{~km} \mathrm{~s}^{-1}$ 

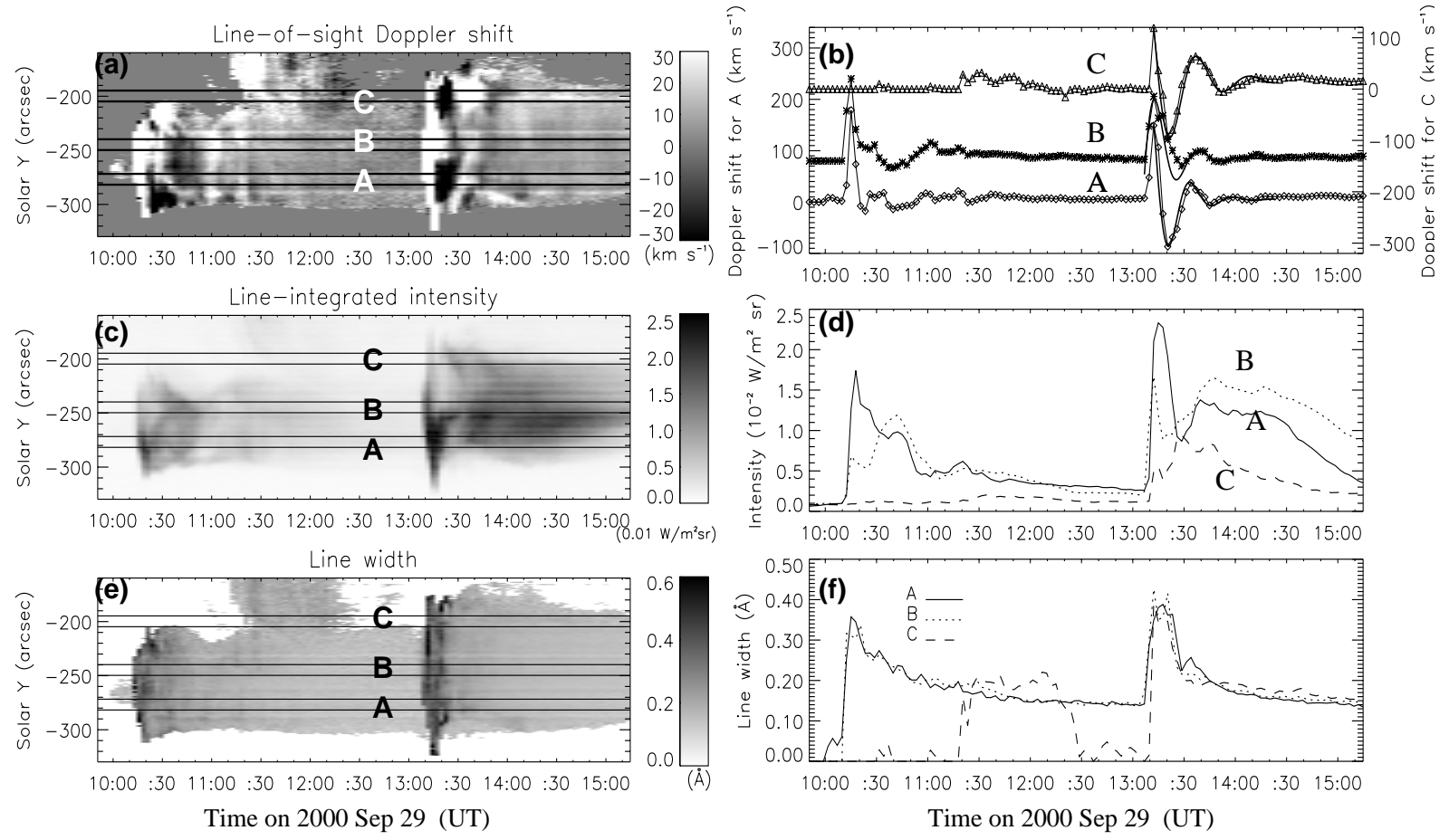

Fig. 7. Doppler oscillation events (Nos. 4A, 4B, 4C) in the Fe XIX line on 29 September 2000. In b) the curve B is plotted relative to the left $y$-axis, but shifted $80 \mathrm{~km} \mathrm{~s}^{-1}$ in order to be seen clearly.

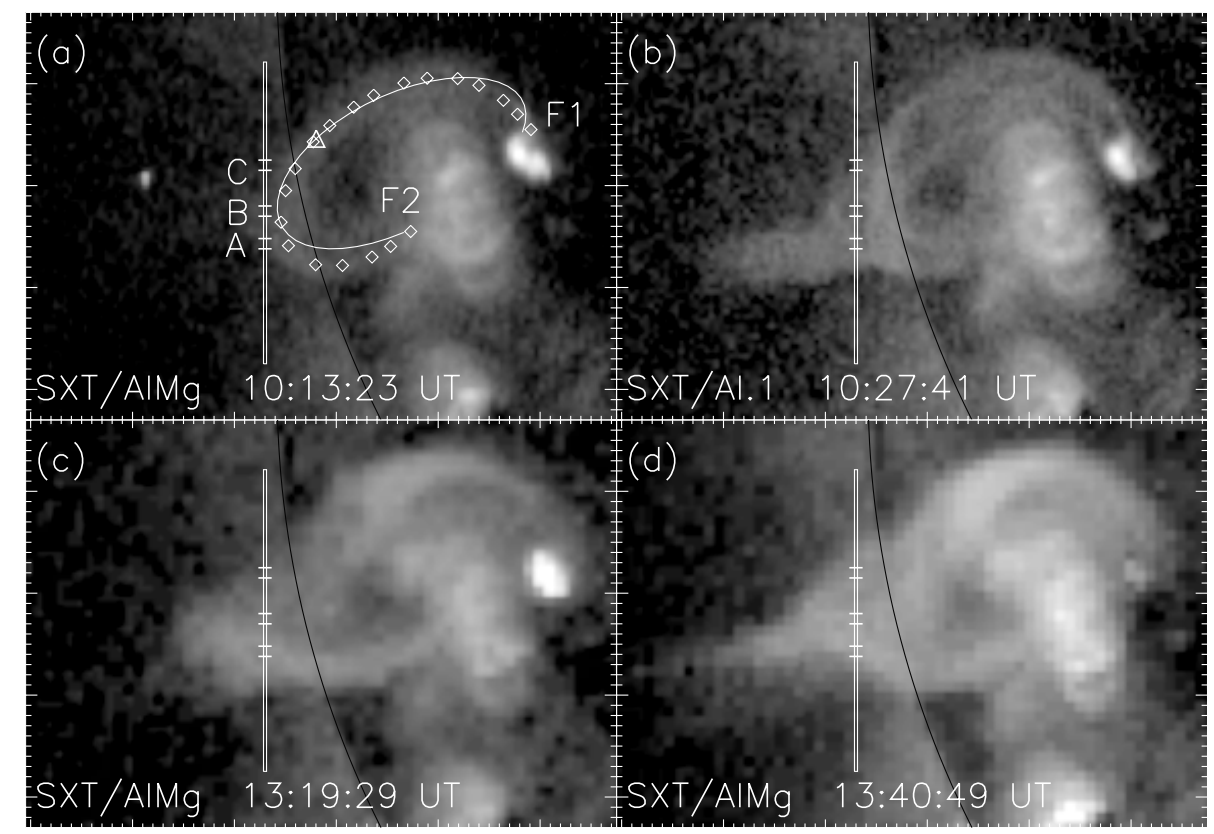

Fig. 8. a) The oscillating soft X-ray loop (outlined with diamonds) fitted with an elliptical model (white curve) for the 29 September 2000 events. The apex position of the modelled loop is marked with a triangle. The SUMER spectrometer slit position is indicated, and positions of three cuts (denoted A, B and C in Fig. 7) are marked. b) The SXT image at 10:27:41 UT. c) The SXT image at 13:19:29 UT. d) The SXT image at 13:40:49 UT.

from cuts 2, 4 and 6 . It is, however, unclear whether there really is a disturbance propagating from $C$ to $B$ and $D$, or if these cuts represent oscillations of different loops crossing the slit at these points (inferred from different periods, phases, and decay rates). Figures $9 \mathrm{e}$ and $9 \mathrm{f}$ show that variations of the line width are also distinctly different along the slit (e.g. at $B, C$, and $D$ ) in the same event.

\subsection{April 2002}

This example shows two oscillation events occurring within 2 hours at the east limb (Fig. 10). No GOES flares were associated with these events. During the first event, the oscillations have periods in the range 16-19.4 min, with decay times of 10-19 min. This event began with strong red-shifts of more 
(a) Line-of-sight Doppler shift

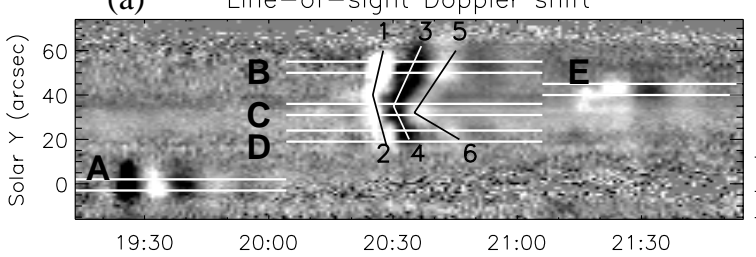

(c)

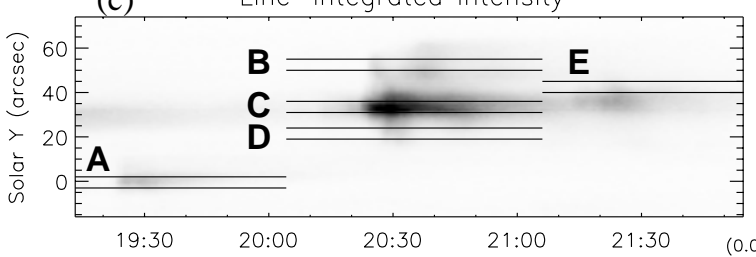

(e)

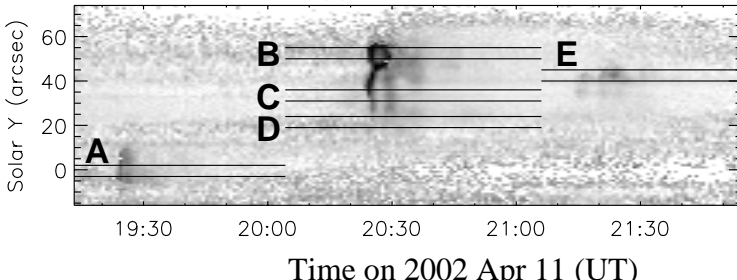

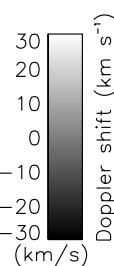
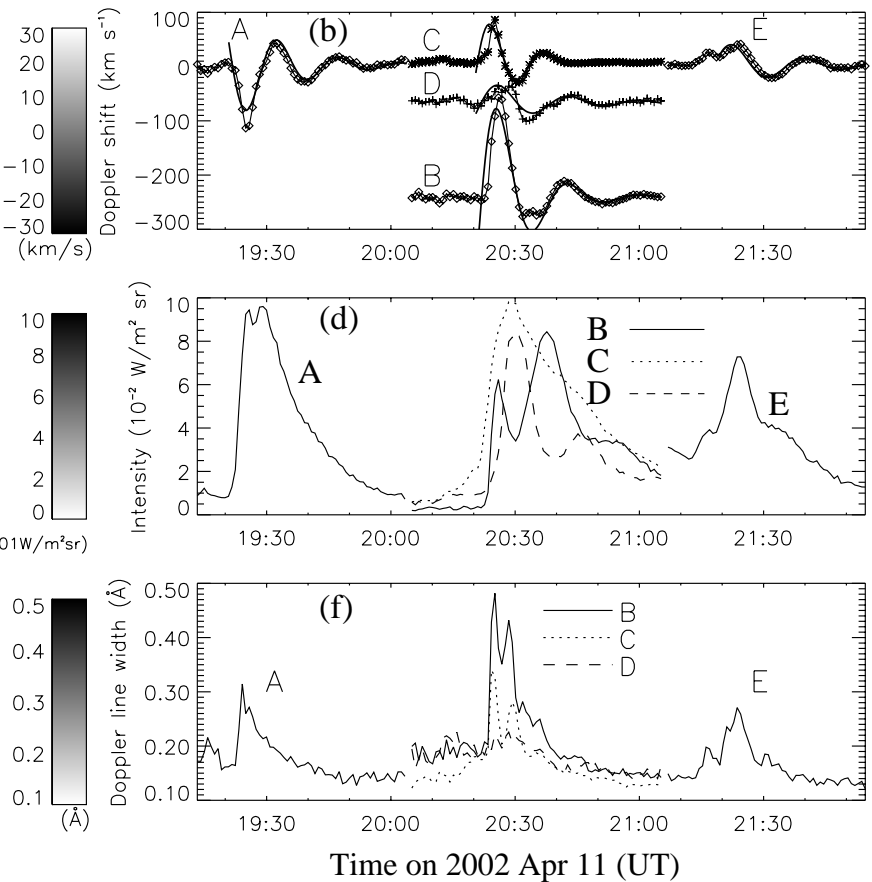

Fig. 9. Doppler oscillation events (Nos. 10A, 11B, 11C, 11D, 12E) detected in the Fe XIX line on 11 April 2002. In b) the curve B is plotted after shifting by $-240 \mathrm{~km} \mathrm{~s}^{-1}$ along the $y$-axis, curve D by $-70 \mathrm{~km} \mathrm{~s}^{-1}$ in order to enhance clarity. In d) the time profiles for A, B, D and E have been stretched by a factor of 5 to facilitate comparison.

(a) Line-of-sight Doppler shift

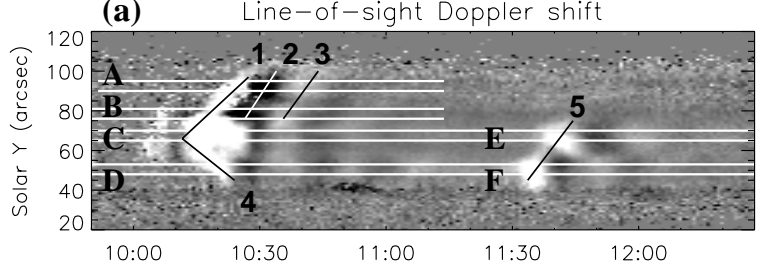

(c) Line-integrated intensity
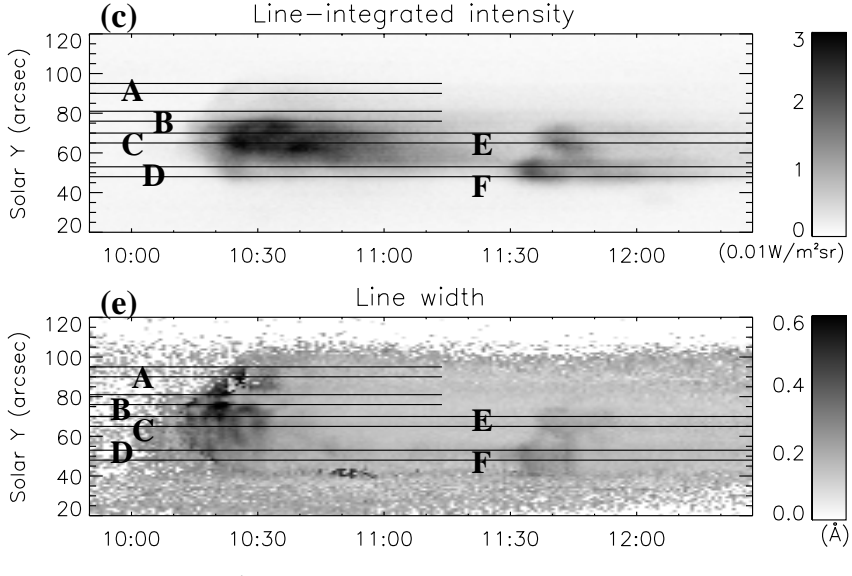

Time on 12 Apr 2002 (UT)
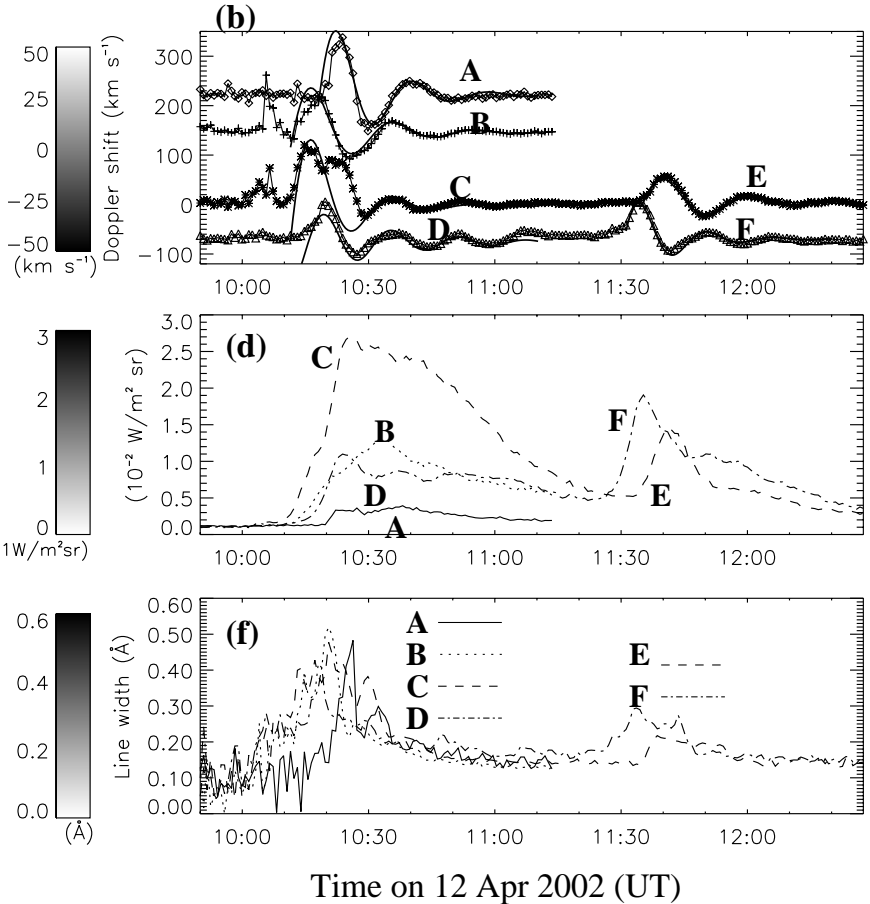

Fig. 10. Doppler oscillation events (Nos. 15A, 15B, 15C, 15D, 16E, 16F) seen in the Fe XIX line on 12 April 2002. In b) the curves A, B and $\mathrm{D}+\mathrm{F}$ are plotted with shifts of $220,150,-70 \mathrm{~km} \mathrm{~s}^{-1}$ along the $y$-axis, respectively.

than $100 \mathrm{~km} \mathrm{~s}^{-1}$ at position $C$ along the slit, and showed distinct propagation along the slit towards the north and south. We measure the speed of these phase propagations from $C$ to $A$ as 24,38 , and $35 \mathrm{~km} \mathrm{~s}^{-1}$ (the slopes of cuts $1,2,3$ ), and the speed from $C$ to $D$ as $20 \mathrm{~km} \mathrm{~s}^{-1}$ (the slope of cut 4). The oscillation component $C$ shows that the initial red-shift pulse lasted to the time of blue-shifts at $B$ and $D$. This long duration of the initial pulse brings it out of phase with the remaining oscillation (Figs. 10a and 10b). Obviously, component $C$ may contain an extra part not belonging to the oscillation, possibly corresponding to the triggering outflow. We also notice that the emission of component $C$ is strongest along the slit, 

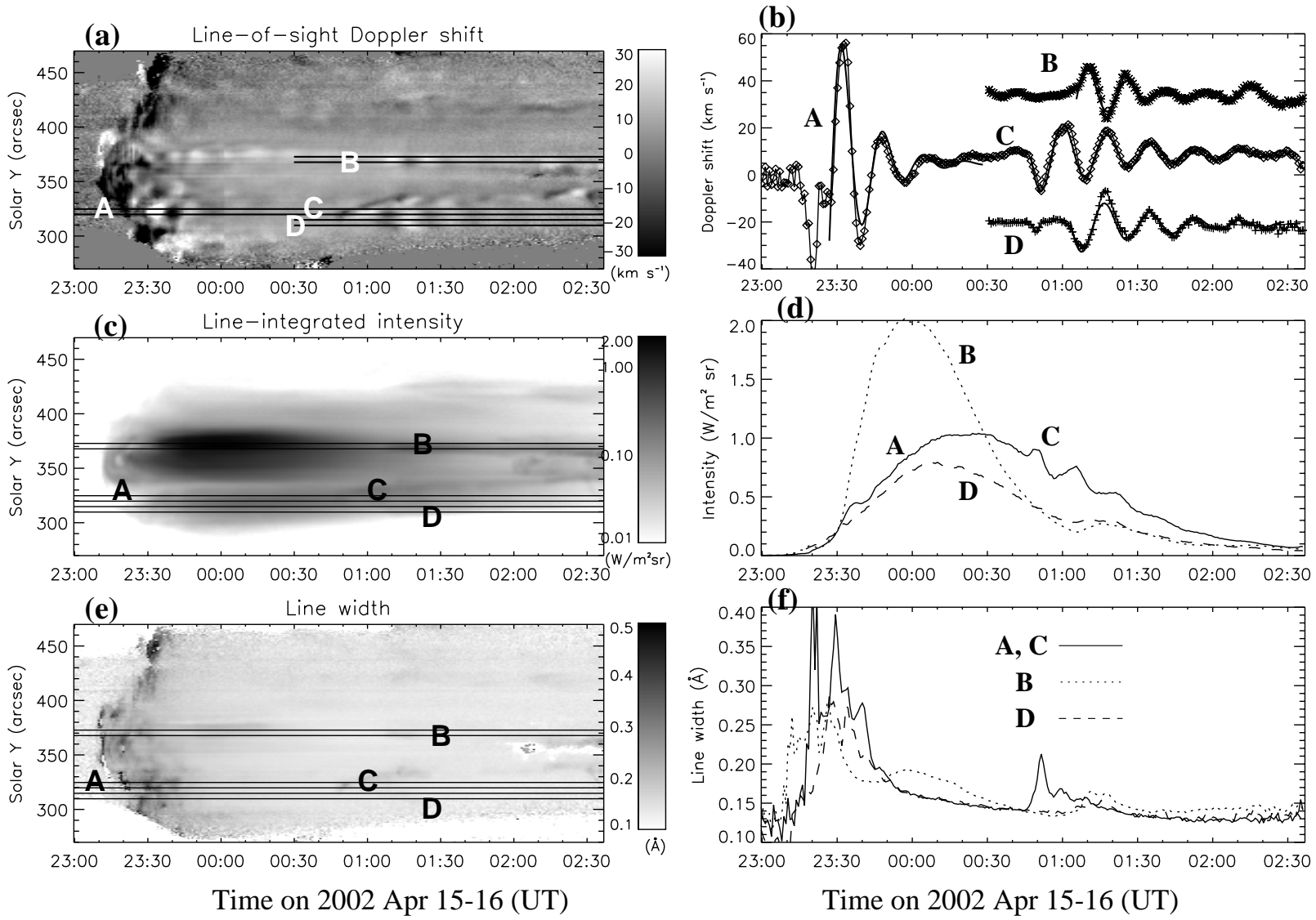

Fig. 11. Doppler oscillation events (Nos. 17A, 18B, 18C, 18D) recorded in the Fe XIX line on 15-16 April 2002. In b) the curves B and D are plotted with shifts of 20 and $-30 \mathrm{~km} \mathrm{~s}^{-1}$ along the $y$-axis, respectively. In c) the brightness is plotted on a logarithmic scale. In d) the temporal profiles for $\mathrm{A}+\mathrm{C}$ and $\mathrm{D}$ have been stretched by a factor of 5 .

showing the phase propagations from the strong emission region towards the fainter ones, which is similar to the behaviour exhibited by the second event on 11 April 2002 (see Sect. 3.4).

The second event shows two neighboring oscillations $(E$ and $F$ ) with an interesting feature. The oscillation $E$ is delayed by half a period compared to oscillation $F$, so that they are in anti-phase. The periods for $E$ and $F$ are $20 \mathrm{~min}$ and $16 \mathrm{~min}$, consistent with those for $C$ and $D$ at the same region along the slit, respectively. The line intensity and width peaks in $E$ also lag behind $F$ (Figs. 10d and 10f). The speed of phase propagation from $F$ to $E$ is about $33 \mathrm{~km} \mathrm{~s}^{-1}$ measured for the initial red-shifts (the slope of cut 5). However, looking carefully at Figs. 10a and 10c the two oscillations appear to be distinct.

\subsection{5-16 April 2002}

This example displays the clearest intensity fluctuations with the same period as the Doppler oscillations and has been analyzed in detail by Wang et al. (2003). Figure 11 shows that several oscillations occurred during a GOES M1.2 flare at the north-west limb. The flare began at 23:05 UT and peaked at 23:24 UT on 15 April in GOES X-ray flux. The total flux in the Fe XIX line over the slit in SUMER shows that this event began at 23:08 UT, and peaked at 23:56 UT. SOHO/EIT in $195 \AA$ channel showed an initial brightening at 23:12 UT in the active region AR 9893, which was followed by an ejection. The oscillation case $A$ occurred in the rising phase of the intensity, with a period of $16.4 \mathrm{~min}$ and a decay time of $12.6 \mathrm{~min}$. The cases $B, C$ and $D$ occurred in the decay phase of the flare, but could be related to small aftermath events at 00:50 UT (for $C$ ) and at 01:10 UT (for $B$ and $D$ ), as indicated in variations of the line intensity and width (Figs. 11d and 11f).

In particular, the oscillations composing case $C$ are distinctly seen for 5 periods, longer than any other oscillation detected by SUMER. The period is $17.6 \mathrm{~min}$ and the decay time is $36.8 \mathrm{~min}$. This is an unusual case of oscillations that damps slowly compared to the others. Case $C$ also shows phase propagations of its initial blue- and red-shifts towards the north with a speed of about $8 \mathrm{~km} \mathrm{~s}^{-1}$. Figure $11 \mathrm{~d}$ reveals intensity fluctuations seen for several periods. By subtracting a smooth background trend, the damped intensity oscillations are shown even more clearly. Wang et al. (2003) measured this difference profile and obtained an oscillation period of $17.1 \mathrm{~min}$, and a decay time of $21.0 \mathrm{~min}$. Strikingly, they found a phase difference of exact 1/4-period between the shift and intensity oscillations, characteristic of compressive (slow-mode) standing waves. In addition, we notice that case $C$ shows also periodic fluctuations in line widths (Fig. 11f). But because the period (about $10 \mathrm{~min}$ ) is distinctly smaller than that of the Doppler or intensity oscillation, we exclude the possibility that this line width fluctuation is caused by the slow waves (i.e. relevant temperature disturbances). Instead it may infer a periodic heating. 
Table 4. Average and range of physical parameters of 54 Doppler-shift oscillations.

\begin{tabular}{lrr}
\hline \hline Parameters & Average & Range \\
\hline Oscillation period $P$ & $17.6 \pm 5.4 \mathrm{~min}$ & $7.1-31.1 \mathrm{~min}$ \\
Decay time $\tau_{\mathrm{d}}$ & $14.6 \pm 7.0 \mathrm{~min}$ & $5.7-36.8 \mathrm{~min}$ \\
Doppler oscillation amplitude $V_{\mathrm{D}}$ & $98 \pm 75 \mathrm{~km} \mathrm{~s}^{-1}$ & $14-319 \mathrm{~km} \mathrm{~s}^{-1}$ \\
Maximum Doppler amplitude $V_{\mathrm{m}}$ & $75 \pm 53 \mathrm{~km} \mathrm{~s}^{-1}$ & $11-234 \mathrm{~km} \mathrm{~s}^{-1}$ \\
Derived displacement amplitude $A$ & $12.5 \pm 9.9 \mathrm{Mm}$ & $1.7-43.7 \mathrm{Mm}$ \\
Ratio of decay time to period $\tau_{\mathrm{d}} / P$ & $0.85 \pm 0.35$ & $0.33-2.1$ \\
Number of periods $N_{\mathrm{P}}$ & $2.3 \pm 0.7$ & $1.5-5$ \\
Time lag of intensity peak $\Delta T_{\mathrm{IV}}$ & $8.5 \pm 13.1 \mathrm{~min}$ & $-2.5-52.5 \mathrm{~min}$ \\
Time lag of line width peak $\Delta T_{\mathrm{WV}}$ & $1.0 \pm 3.0 \mathrm{~min}$ & $-4.1-9.1 \mathrm{~min}$ \\
Intensity peak duration $\Delta T_{\mathrm{I}}$ & $36.2 \pm 27.0 \mathrm{~min}$ & $10-141 \mathrm{~min}$ \\
Number of intensity peaks $N_{\mathrm{I}}$ & $1.5 \pm 0.7$ & $1-3$ \\
Speed of phase propagation & $43 \pm 25 \mathrm{~km} \mathrm{~s}^{\mathrm{a}}$ & $8-102 \mathrm{~km} \mathrm{~s}^{-1}$ \\
Spatial extent of osci. along slit $\Delta Y$ & $35 \pm 21 \mathrm{Mm}$ & $7-87 \mathrm{Mm}$ \\
\hline
\end{tabular}

a This quantity is measured only for the cases with a clear phase propagation (see Sect. 4).

\section{Statistical results}

We analyzed 54 Doppler shift oscillations associated with 27 flux enhancement events of hot plasma. The oscillatory Doppler shifts happened in regions extending 7-87 Mm along the slit with a mean of $35 \pm 21 \mathrm{Mm}$ (Table 4 ). In each region, we identified several oscillation components along the slit, due to differences in period or phase. The physical parameters obtained from the time series analysis are listed in Tables 1 and 4 and their statistical distributions are shown in Figs. 12 and 13. The results are summarized as follows.

For all cases the Doppler oscillations were detected only in flare lines $(T=6-10 \mathrm{MK})$. The oscillations were seen more often in the Fe XIX line, than in the Fe XXI line. Only 3 of the 27 events are seen in Fe XXI (see Table 1). For example in April-May 2002, 19 events are observed during an observing time of 18.4 days in Fe XIX, while only 2 events during an observing time of 14.6 days in Fe XXI. This is mainly because impulsive intensity brightenings are detected more often in Fe XIX than in Fe XXI. Since the SUMER detector is equally sensitive to these two lines, this indicates that there is a temperature dependence of the occurrence rate of impulsive intensity brightenings or Doppler oscillation events.

We find that all events show the flux variations of flarelike impulsive profiles, but only 7 of the 27 events were associated with GOES flares (see Table 2). This is in contrast to the transverse loop oscillations seen by TRACE, all of which are associated with flares. It is also significantly lower than the 50\% (i.e. 13-14 events) expected if the X-ray brightening occurs in just one loop footpoint which may be located behind the solar limb. For these flare-associated events, the start times of GOES X-ray flux and of SUMER flare line intensity are consistent. In some events not associated with flares (e.g. events. 15 and 17), SOHO/EIT detected brightenings occurring in the limb active region SUMER aimed at, thus suggesting that the SUMER brightenings may correspond to small flares having an X-ray flux below the detection threshold of GOES. We find that 15 of the 27 events belong to recurring events, i.e. they recur 2-3 times within about 2 hours at the same place and manifest similar oscillation features such as identical periods and initial Doppler shifts of the same sign (see examples in Figs. 2, 5, 9 and 10). These features suggest that the recurring Doppler oscillations are related to the same magnetic structures. In some cases, the coordinated Yohkoh/SXT images show that the Doppler oscillations happen at locations at which the slit crosses the soft X-ray loops (see Figs. 3, 6, and 8), indicative of hot loop oscillations. The geometric parameters of these loops, determined on the basis of an assumed circular or elliptical shape, are given in Table 3.

We find oscillation periods in the range 7.1-31.1 min with a mean of $17.6 \pm 5.4$ min (Tables 1 and 4). Although the observations with a high cadence of $50 \mathrm{~s}$ allow a detection of short-period oscillations of $\sim 3$ min period, almost all cases have periods larger than $10 \mathrm{~min}$ (Fig. 12a), except for a peculiar case $27 \mathrm{~B}$. The event 27 occurred in the southwest limb, and was associated with a M1 flare (Table 2). This event showed strong initial red shifts up to $100 \mathrm{~km} \mathrm{~s}^{-1}$ (Fig. B.11 in Appendix B). Case $27 B$ has the shortest period of 7.1 min of all studied cases. That the high cadence observations do not show shorter oscillations suggests that the cutoff at higher frequency is not a bias introduced by instrumental concerns (although the fact that the slit was always placed a certain distance above the limb may play a role, since only the larger loops are sampled). The statistic distribution shows that the Doppler oscillations have periods much longer than the transverse loop oscillations ( $P=2-11 \mathrm{~min}$ ) observed by TRACE (Fig. 12a). Compared to the propagating longitudinal waves $(P=2.4-8.6 \mathrm{~min})$ found in the footpoints of the TRACE loops (De Moortel et al. 2002a), the periods of the SUMER loop oscillations are also distinctly longer.

The Doppler oscillations suffer from very strong damping and are generally visible only for 2 or 3 periods. The average number of observed periods is $2.3 \pm 0.7$ (Table 4 ), about half 

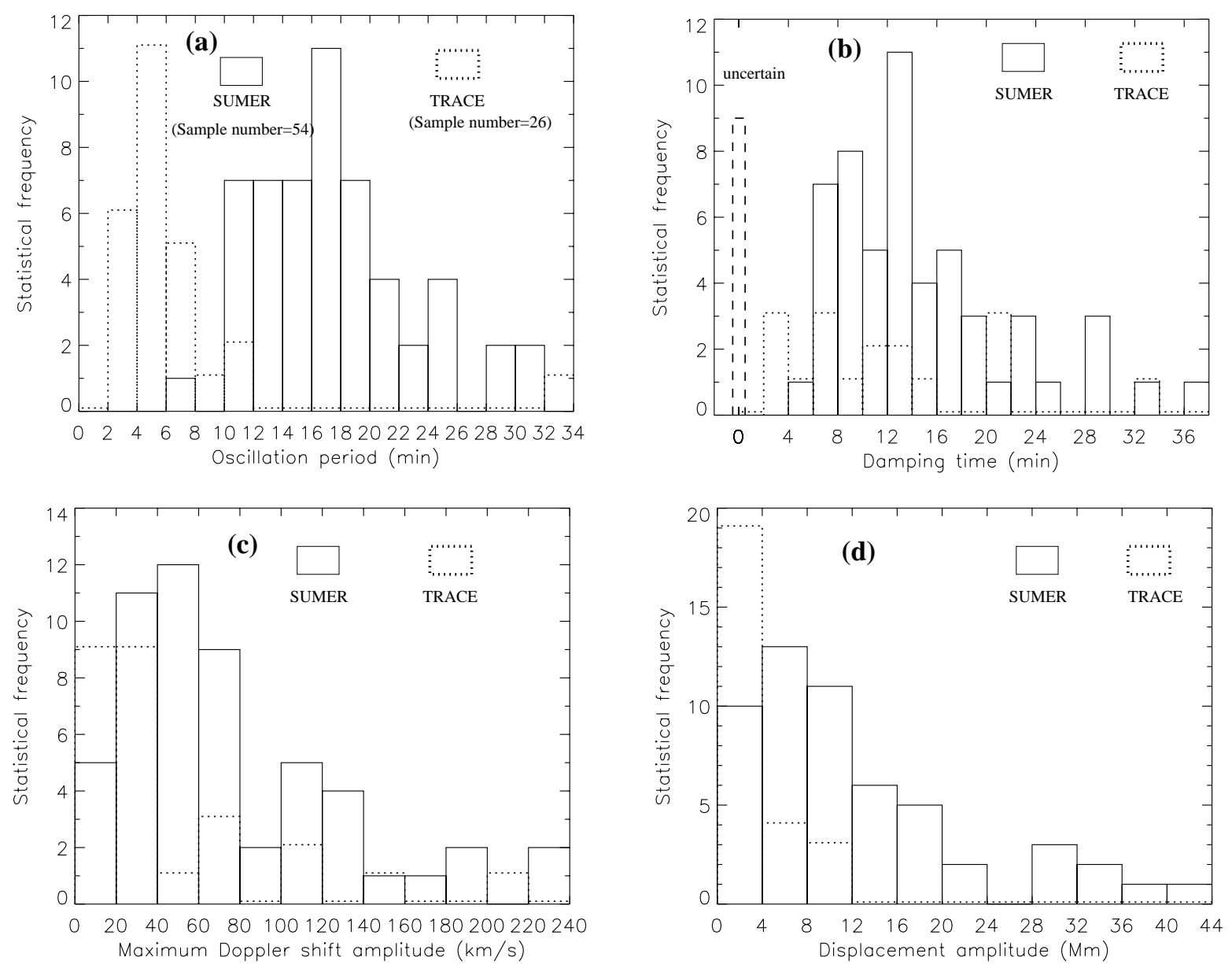

Fig. 12. Distribution of the physical parameters of the 54 SUMER Doppler-shift oscillations (solid histograms), and distribution of the parameters of the 26 TRACE transversal loop oscillations (dotted histograms) obtained by Aschwanden et al. (2002). a) Oscillation periods. b) Damping time. The number of TRACE loop oscillations whose damping time could not be evaluated is represented with a dashed strip. c) Measured maximum Doppler velocity amplitude for SUMER oscillations, and the maximum transverse speed for TRACE oscillations. d) Derived displacement amplitude for SUMER oscillations, and the transverse motion amplitude for TRACE oscillations.

of the number $(4.0 \pm 1.8)$ seen in TRACE data (Aschwanden et al. 2002). We find decay times in the range 5.7-36.8 min, with a mean of $14.6 \pm 7.0 \mathrm{~min}$. The ratio of the decay time to the period is $0.85 \pm 0.35$ (Fig. 13d), which is about a factor of 2 shorter than that $(\sim 1.8)$ of TRACE oscillations. A comparison of the distributions of decay times obtained for the SUMER and TRACE oscillations is shown in Fig. 12b. The weakest damping is observed in case $18 C$ (Fig. 11). In this case the oscillations lasted for 5 periods with the decay time being about twice the period. This is also the clearest case showing a damped intensity oscillation. It damps faster than the Doppler shift oscillation (see Sect. 3.6 and Wang et al. 2003). Case $2 A$ provides another example that shows the damped intensity oscillation associated with the Doppler oscillation (Fig. 14). For cases $21 B$ (Fig. B.6) and 25A (Fig. B.10) with large initial velocities and less than 2 periods visible in all, we obtain the smallest ratio of decay time to period of about 0.3 (Fig. 13d). In addition, for cases $27(A, B, C)$ (Fig. B.11), a peculiar behavior is observed, with 1.5 periods of slowly damped oscillations followed by a subsequent rapid decay. Hence measurements of the decay time for these cases $(21 B, 25 A$ and $27[A, B, C])$ have a large error. Excluding these 5 dubious cases, we find that the scaling of the decay time with the period for the remaining 49 cases is

$\tau_{\mathrm{d}}=0.68_{-0.27}^{+0.46} P^{1.06 \pm 0.18}$

with a correlation coefficient of 0.66 (Fig. 15). This result agrees well with that $\left(\tau_{\mathrm{d}}=0.61 P^{1.07 \pm 0.16}\right)$ obtained on the basis of data for 35 cases by Wang et al. (2002c). Including all cases, the correlation coefficient decreases to 0.54 , so that the obtained scaling is less reliable:

$\tau_{\mathrm{d}}=1.40_{-0.54}^{+0.88} P^{0.80 \pm 0.17}$.

We find that the oscillations have an initial large Doppler shift pulse with peak velocities up to $200 \mathrm{~km} \mathrm{~s}^{-1}$ (e.g. cases $19 \mathrm{~A}$ in Fig. B.4b and $21 B$ in Fig. B.6b in Appendix B). The maximum Doppler shift amplitudes are measured from the data, with a mean and standard deviation of $V_{\mathrm{m}}=75 \pm 53 \mathrm{~km} \mathrm{~s}^{-1}$ (Tables 1 and 4). If we take the sound speed $c_{\mathrm{s}}=380 \mathrm{~km} \mathrm{~s}^{-1}$ corresponding to the formation temperature of Fe XIX ( $T=$ $6.3 \mathrm{MK})$, we obtain a subsonic Mach number of $M=V_{\mathrm{m}} / c_{\mathrm{s}}=$ $0.20 \pm 0.14$. The maximum displacements are derived by $A=$ $V_{\mathrm{m}} /\left(\omega^{2}+\lambda^{2}\right)^{1 / 2}$, with a mean of $12.5 \pm 9.9 \mathrm{Mm}$. Compared 

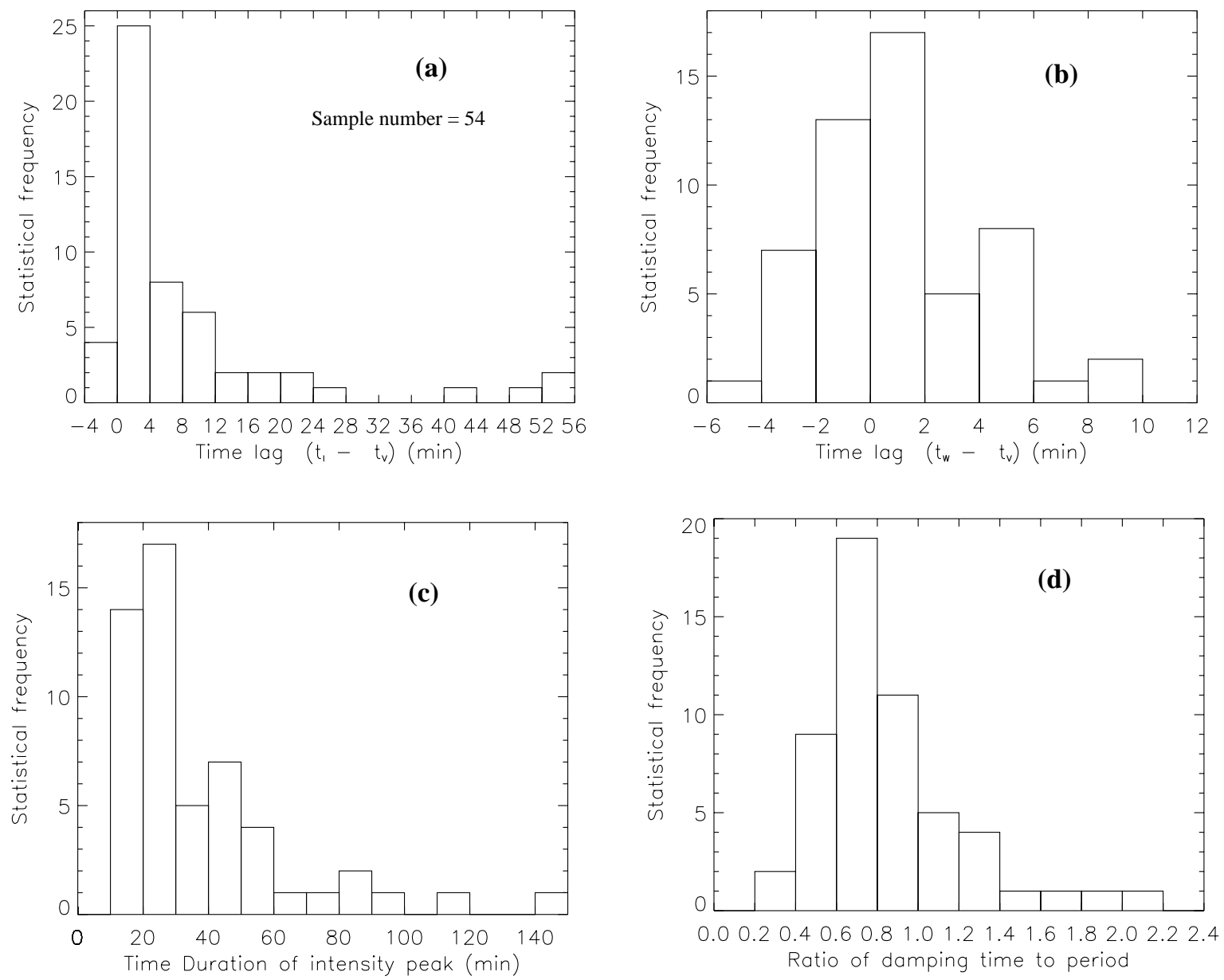

Fig. 13. Distribution of the physical parameters of the 54 SUMER Doppler-shift oscillations. a) Time lag of the maximum intensity peak to the maximum Doppler-shift pulse. b) Time lag of the maximum Doppler line width peak to the maximum Doppler-shift pulse. c) Duration of the intensity peak. d) Ratio of the damping time to the period.

to the TRACE transverse loop oscillations, the velocity amplitudes are roughly the same (Fig. 12c), but the derived displacement amplitudes are distinctly larger by a factor of 4 or 5 times (Fig. 12d).

The Doppler shift generally peaks earlier than the intensity, but almost simultaneously with the line width (Figs. 13a and 13b). The average time lag of the maximum intensity peak to the maximum Doppler shift pulse is $8.5 \pm 13.1 \mathrm{~min}$, and that of the maximum line width peak to the maximum Doppler shift pulse is $1.0 \pm 3.0 \mathrm{~min}$ (Tables 1 and 4 ). The duration of the intensity peak (defined as the time spent at brightness above $1 / e$ of the maximum) is on average $36.2 \pm 27.0 \mathrm{~min}$, about twice the average period (Fig. 13c and Table 4). In 22 of 54 cases, the intensity profiles have multiple peaks with a mean number of $1.5 \pm 0.7$. But with the exception of cases $2 A$ and $18 C$, which show intensity fluctuations with the same period as the Doppler oscillation, the presence of a definite period is not so certain in the other cases.

We find that neighboring components along the slit oscillate in anti-phase in 5 cases $(5[A, B], 6[C, D]$ in Fig. 2, $16[E, F]$ in Fig. $10,18[B, C]$ in Fig. 11 , and $8[B, C]$ in Fig. B.1 in Appendix B). The two components are $15^{\prime \prime}-50^{\prime \prime}$ apart, with a mean of $24^{\prime \prime} \pm 13^{\prime \prime}$. In 3 out of 5 cases (i.e. $5[A, B]$,

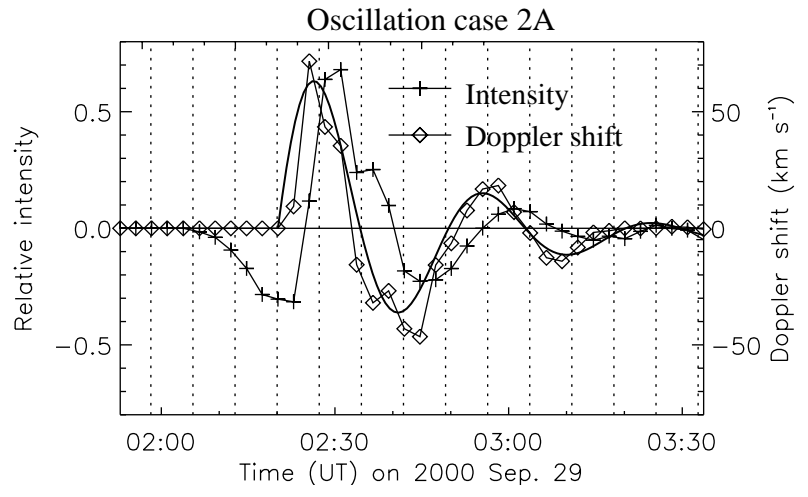

Fig. 14. Doppler and intensity oscillations in the FeXIX line for case 2A. The thick solid curve is the best fit to the Doppler shifts with a damped sine function. For the intensity curve, an 11-pixel smooth background trend has been subtracted, and the variation is normalized relative to the maximum value of the background trend.

$6[C, D]$, and $8[B, C])$, these oscillations start simultaneously, while there is a phase decay in cases $16[E, F]$ ( 0.5 period) and $18[B, C]$ (1 period). The phase relations of these neighboring oscillations suggest that their corresponding magnetic structures (or coronal loops) are related. Oscillations in 


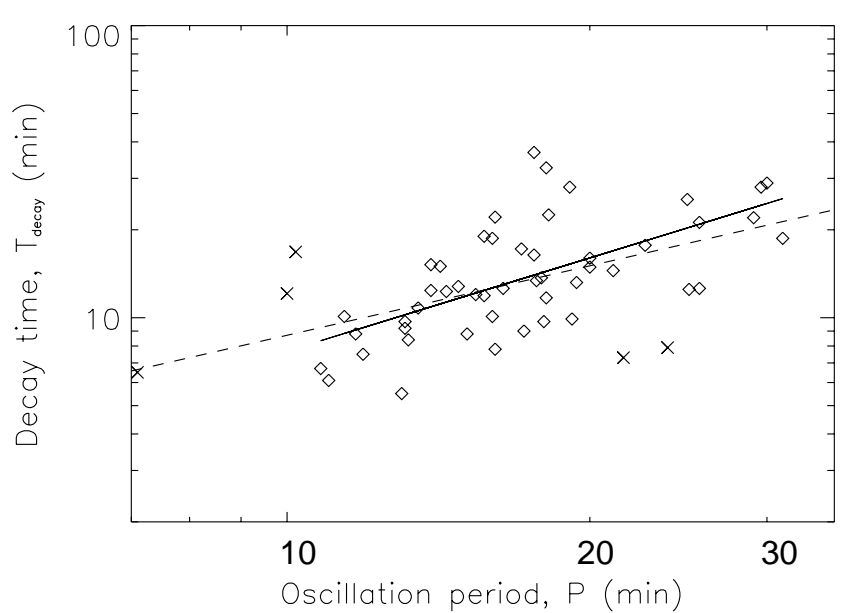

Fig. 15. Scaling of the damping time of Doppler oscillations with the period. The solid line is the best fit scaling for the 49 oscillations (diamonds) excluding the 5 dubious cases (crosses) (i.e. $21 B$, 25A, and $27(A, B, C))$. The dashed line is the best fit scaling for all cases.

antiphase have been detected in neighbouring loops by TRACE (Schrijver et al. 2002).

The high cadence observations reveal phase propagation along the slit in some oscillations. In events 11,15 , and 18 (see the examples in Sects. 3.4, 3.5, and 3.6), we have found that the alternating blue- and red-shifts show an increasing phase delay along the slit in one or in both directions when starting from a particular point, with propagation speeds in the range $8-96 \mathrm{~km} \mathrm{~s}^{-1}$. This feature is also seen in events 7, 19 and 20. For most of the cases, the region with the strongest emission oscillates first, with fainter regions showing an increasing delay. For event 7, we measure speeds of 24 and $31 \mathrm{~km} \mathrm{~s}^{-1}$ from cuts 1 and 2 along the slit (Fig. B.1a). For event 19 , the speeds are 51,33 , and $37 \mathrm{~km} \mathrm{~s}^{-1}$ from cuts 1 , 2 and 3 (Fig. B.4a). For event 20, the speeds are 36, 34, 102 and $57 \mathrm{~km} \mathrm{~s}^{-1}$ from cuts 1, 2, 3 and 4 (Fig. B.5a). For 20 measurements of the phase propagation in 6 events, we obtain an average speed of $43 \pm 25 \mathrm{~km} \mathrm{~s}^{-1}$ along the slit (Table 4). In addition, case 24A shows that the oscillatory region moves southward with time along the slit at a speed of $6 \mathrm{~km} \mathrm{~s}^{-1}$ (Fig. B.9a).

\section{Discussion}

\subsection{Oscillations in the slow standing mode}

We now investigate the physical mechanism of hot loop oscillations observed by SUMER. Under coronal conditions (where the Alfvén velocity $v_{\mathrm{A}}$ is much larger than the sound speed $c_{\mathrm{s}}$ ), there are two, well-separated, modal classes of oscillations of a magnetic flux tube, corresponding to the usual fast and slow magnetoacoustic waves (Roberts et al. 1984). The fast waves (the sausage and kink modes) have a phase speed of $\omega / k \geq v_{\mathrm{A}}$, and the slow waves have a phase speed of $\omega / k \simeq c_{\mathrm{t}}$, where the tube speed $c_{\mathrm{t}}=c_{\mathrm{s}} v_{\mathrm{A}} /\left(c_{\mathrm{s}}^{2}+v_{\mathrm{A}}^{2}\right)^{1 / 2} \simeq c_{\mathrm{s}}$. The transverse oscillations of the comparatively cool loops observed by TRACE have been interpreted in terms of the standing kink mode (Aschwanden et al. 1999b, 2002; Nakariakov et al. 1999). Our observations show that the hot loop oscillations have much longer periods and larger amplitudes than the TRACE loop oscillations. The scaling of the decay time with the period is also different in the cool and hot loops (Ofman \& Aschwanden 2002; Ofman \& Wang 2002). These facts suggest a different wave mode and a different damping dissipation mechanism in the cool and hot loops (Wang et al. 2002c; Ofman \& Wang 2002).

Based on an analysis of oscillation $18 C$ on 16 April 2002, Wang et al. (2003) have demonstrated that the hot loop oscillations detected by SUMER belong to the slow standing mode, at least in this particular case. Here we discuss the full set of observations in the light of this result.

Whether a slow-mode wave is a propagating or standing wave can be inferred from the phase relationship between velocity and intensity: propagating waves give an in-phase variation, whereas standing waves give a $1 / 4$-period phase difference (Sakurai et al. 2002). Thus, based on an in-phase relationship with the line intensity, Sakurai et al. (2002) identified the Doppler oscillations observed in the coronal green line (Fe XIV, 2 MK) $5303 \AA$ as propagating slow waves. The periodic intensity fluctuation seen in case $18 C$, has the same period as the Doppler oscillation and a time lag of exactly $1 / 4$-period. Thus, these observations indicate the presence of slow standing waves in hot coronal loops. This conclusion is supported by a modeling result in Ofman \& Wang (2002).

Case $18 C$ shows that the intensity oscillation damps more rapidly than the Doppler oscillation. This implies that oscillatory signals in intensity are more difficult to detect. Case $18 \mathrm{C}$ is exceptional in some ways (slow damping, smoothly varying background intensity) which allow the intensity fluctuations to be easily extracted. For most other cases, Doppler oscillations usually have a decay time on the order of the period $\left(\tau_{\mathrm{d}} / P \sim 1\right)$ and only a few periods are visible $\left(N_{\mathrm{P}} \sim 2\right)$. At the same time the intensity also changes rapidly due to the generally short-lived brightening of Fe XIX or Fe XXI emission. Hence, although nearly half of all cases show several peaks in the intensity profile, it is difficult to separate the evolution of the brightening from a damped periodic signal. Nevertheless, for case $2 A$ it has also been possible to extract the intensity oscillations. We find that case $2 A$ also shows a roughly $1 / 4$-period phase relationship between the Doppler and intensity oscillations (Fig. 14).

In several cases, the geometric parameters of oscillating loops, especially the loop length, are determined from the Yohkoh/SXT images (Table 3), allowing us to further examine the possible mode. If the slow waves occur as standing modes in a loop of length $L$, their period is given by (Roberts et al. 1984),

$\tau_{\mathrm{s}}=\frac{2 L}{j c_{\mathrm{t}}} \approx \frac{2 L}{j c_{\mathrm{s}}}$

with the sound speed $c_{\mathrm{s}} \simeq 1.5 \times 10^{4} T^{1 / 2}$.

For the events observed on 9 March 2001, occurring in a loop of length $L=140 \mathrm{Mm}$ with temperature $T=6.3 \mathrm{MK}$ (appropriate for Fe XIX and giving a sound speed of $c_{\mathrm{s}}=$ $380 \mathrm{~km} \mathrm{~s}^{-1}$ ), the slow mode with $j=1$ (i.e. no node along the loop) produces a period of $\tau_{\mathrm{s}}=12 \mathrm{~min}$. This is very close to the observed periods of 14-18 $\mathrm{min}$. In another case 
on 29 September 2000 for a loop of $L \simeq 500 \mathrm{Mm}$ with $T=$ $6.3 \mathrm{MK}$ and $j=1$, we obtain $\tau_{\mathrm{s}}=44 \mathrm{~min}$. This is $1.4-1.8$ times the observed periods $(P=25-31 \mathrm{~min})$. For $j=2$ we get $\tau_{\mathrm{s}} \simeq 22 \mathrm{~min}$ which lies close to the observed range of periods. Because the loop baseline (assumed parallel to the loop top) has an angle to the line-of-sight of $\psi=34^{\circ}-58^{\circ}$ (Table 3), significant Doppler oscillations are expected to be seen for the compressive slow modes $\left(v_{\text {obs }}=v_{\text {true }} \cos \psi \simeq(0.8-0.5) v_{\text {true }}\right)$. These estimates support the interpretation.

Consider now the alternative that the oscillations are transverse. In a uniform magnetic field the period of the standing kink mode in its fundamental mode is (Roberts et al. 1984),

$\tau_{\mathrm{k}}=\frac{2 L}{c_{\mathrm{k}}}, \quad c_{\mathrm{k}}=v_{\mathrm{A}}\left(\frac{2}{1+n_{\mathrm{e}} / n_{0}}\right)^{1 / 2}$,

where $n_{0}$ and $n_{\mathrm{e}}$ are the plasma densities inside and outside the loop, respectively. If we require that the period of the kink mode matches the observed period, i.e. $\tau_{\mathrm{k}} \simeq P$, then the Alfvén velocity can be derived from Eq. (3),

$v_{\mathrm{A}}=\frac{\sqrt{2} L}{P}\left(1+\frac{n_{\mathrm{e}}}{n_{0}}\right)^{1 / 2} \simeq 1.5 \frac{L}{P}$,

where we assume $n_{\mathrm{e}} / n_{0} \simeq 0.1$. For the 9 March 2001 case with the average period $P \simeq 16 \mathrm{~min}$ and $L=140 \mathrm{Mm}$, we obtain $v_{\mathrm{A}}=219 \mathrm{~km} \mathrm{~s}^{-1}$, from which we deduce that the plasma $\beta[\equiv$ $\left.(2 / \gamma)\left(c_{\mathrm{s}}^{2} / v_{\mathrm{A}}^{2}\right)\right] \simeq 3.6$. For the 29 September 2000 case with $P \simeq$ $28 \mathrm{~min}$ and $L=500 \mathrm{Mm}$, we have $v_{\mathrm{A}}=446 \mathrm{~km} \mathrm{~s}^{-1}$ and $\beta \simeq 0$.9. Therefore, an interpretation of the oscillations in terms of the kink mode requires a plasma $\beta$ of order unity, implying an unusual coronal loop environment. For example, studies of coronal loops in active regions on the basis of X-ray data give the $\beta$ values in the range $10^{-5}-10^{-2}$ (e.g. Schmelz et al. 1994; McKenzie \& Mullan 1997). However, Gary (2001) argued that the plasma $\beta$ can increase beyond unity at relatively low coronal heights $\left(h \sim \frac{1}{4}-\frac{3}{4} R_{\odot}\right)$. We derive a loop height of $h \simeq$ $40 \mathrm{Mm}$ in the 9 March 2001 case and $h \simeq 100 \mathrm{Mm}$ in the 29 September 2000 case. According to the plasma beta model by Gary (2001), $0.005<\beta<0.1$ at $h \simeq 40 \mathrm{Mm}$ and $0.04<\beta<$ 0.4 at $h \simeq 100 \mathrm{Mm}$. These estimates are also in disagreement with the $\beta$ values derived from the SUMER loop oscillations, and hence do not support the kink mode explanation.

We notice that the derived displacement amplitudes $(A=$ $12.5 \pm 9.9 \mathrm{Mm}$ ) from the shift integration are very large, about 5 times that of the transverse oscillations observed by TRACE. Assuming that the kink mode occurs with an amplitude $A \simeq$ $12 \mathrm{Mm}$ for a loop with an azimuth angle of $\alpha=45^{\circ}$, we obtain the transverse amplitude $A_{\text {trans }}=A \cos \alpha \simeq 8 \mathrm{Mm}$. This amplitude corresponds to about 4 pixels in SXT images with a full-resolution $\left(\sim 2\right.$ 2. $^{\prime} 5$ pixel $\left.^{-1}\right)$, which could be well resolved, but such oscillation cases have never been found in the previous studies of SXT observations (e.g. McKenzie \& Mullan 1997). For slow waves, however, no significant displacements of the loops' magnetic field are expected, in agreement with SXT observations. Considering an interpretation of the SUMER oscillations in terms of the kink mode for a coronal loop with a typical width $w \simeq 9 \mathrm{Mm}$ (Aschwanden et al. 2002) and with an inclination angle of $\theta \simeq 30^{\circ}$ to the vertical (e.g. the cases listed in Table 3), and assuming the loop top to be initially at the slit position, we estimate that the loop top moves away from the slit in the vertical direction by $\Delta x=A \sin \theta \simeq 6 \mathrm{Mm}$, when the loop reaches a kink-mode amplitude of $A=12 \mathrm{Mm}$. This deviation is nearly the size of the loop width, implying that the oscillating loop almost moves off the slit. This should cause a large decrease of line-integrated intensity. Due to the oscillation of the loop, making it periodically pass onto and away from the slit, the line-integrated intensity should manifest periodic fluctuations with a frequency twice that of the Doppler shift oscillations. However, such features were not observed in any of the cases, which also argue against the kink-mode assumption.

Quasi-periodic $(P \simeq 20 \mathrm{~min})$ brightness variations in $>3.5 \mathrm{keV} \mathrm{X}$-rays associated with large coronal loops of length 200-300 Mm were observed by the SMM spacecraft (Švestka et al. 1982; Harrison 1987), and were interpreted in terms of standing slow-mode oscillations ( ̌̌vestka et al. 1994). These long-period pulsations seem to be consistent with the Doppler shift oscillations observed by SUMER, and may be triggered by a similar mechanism, which we will discuss in Sect. 5.3. However, systematic searches for loop oscillations using SXT images by McKenzie \& Mullan (1997) did not show such long-period flux fluctuations, but only short-periodic (9.6-61.6 s) modulations with small amplitudes of about $1 \%$ for coronal loops of length $40-150 \mathrm{Mm}$ in 16 out of 544 cases. But variations with a long period $(P \simeq 20 \mathrm{~min}$ ) could be missed due to the limited durations ( $<31 \mathrm{~min}$ ) of the data records they used, or due to their data selection rules.

\subsection{Damping of oscillations}

Both TRACE and SUMER observations of loop oscillations show strongly decaying modes, raising the question of the cause of this decay. For the transverse Alfvénic oscillations observed by TRACE, proposals for the damping mechanism range from enhanced resistivity or enhanced viscosity due to resonant absorption (Nakariakov et al. 1999) or phase mixing (Ofman \& Aschwanden 2002) to photospheric properties related to nullpoints or separators (Schrijver \& Brown 2000). For the hot loop oscillations observed by SUMER, interpreted as slow-mode waves, the dominant dissipation mechanism (thermal conduction or compressive viscosity) is different from that acting on Alfvén waves (resistivity or shear viscosity). Ofman \& Wang (2002) have modeled the oscillations and the damping of slow standing waves in a model coronal loop for parameters typical of those observed by SUMER, and find that due to the high temperature $(T>6 \mathrm{MK})$ of the loops, the large thermal conduction leads to rapid damping of the slow waves on a timescale comparable to observations. The scaling of the dissipation time with period agrees well with the scaling $\left(\tau_{\mathrm{d}}=0.68_{-0.27}^{+0.46} P^{1.06 \pm 0.18}\right)$ obtained for 49 cases in this study. Ofman \& Wang (2002) also find that the decay time due to compressive viscosity alone is an order of magnitude longer than the observed decay time.

We find that the total duration $\left(\Delta T_{\mathrm{I}}\right)$ of the intensity enhancement is generally about twice as long as the period or the decay time for the hot loop oscillations, and this duration is 
also on the order of the length of time over which the oscillations are visible because the observed number of periods is $N_{\mathrm{p}} \simeq 2.3$. If we assume $\Delta T_{\mathrm{I}}$ to be approximately the cooling time of hot loops, this implies that the thermal conduction dissipation is very efficient within an interval $\Delta T_{\mathrm{I}}$ during which the loops are hot. After this time, the small amplitude of shift oscillations could have been invisible due to the weak emission in Fe XIX and Fe XXI and the limited spectral resolution. For example, for a typical case with $V_{\mathrm{m}}=75 \mathrm{~km} \mathrm{~s}^{-1}$ and $\tau_{\mathrm{d}}=P$, we have $V(t) \simeq 10 \mathrm{~km} \mathrm{~s}^{-1}$ when $t=2 P$, lower than the SUMER spectral resolution $\left(0.0442 \AA /\right.$ pixel $\simeq 12 \mathrm{~km} \mathrm{~s}^{-1}$ in the line Fe XIX $1118 \AA$ A).

Case $18 C$ is a special case which shows the weakest damping with $\tau_{\mathrm{d}} \simeq 2 P$ and 5 clearly visible periods. We notice that this event occurred in the decay phase of a M1.2 flare, so the oscillations could be related to a hot, dense flaring loop, with plasma density possibly 1-2 orders of magnitude larger than the density $\left(\sim 10^{9} \mathrm{~cm}^{-3}\right)$ in a usual AR loop. According to the MHD equations describing a loop, given by Ofman \& Wang (2002), the higher loop density will weaken the damping of oscillations in velocity and temperature, and so may explain the observations.

\subsection{Excitation of oscillations}

In contrast to the TRACE transverse oscillations of cold $(\sim 1 \mathrm{MK})$ loops, the SUMER Doppler shift oscillations are only seen in hot flare lines of $T>6 \mathrm{MK}$. For all cases, impulsive profiles of line-integrated intensity and initial large Doppler broadenings of the lines indicate that the oscillations are excited impulsively. The concurrence of initial strong Doppler shifts and line broadenings suggests that slow-mode waves may be excited by a disturbance with large turbulent velocities possibly associated with a very hot plasma ejection from one footpoint of a coronal loop, the subsequent intensity peak (with a typical time lag of $\Delta T_{\mathrm{IV}}=9 \mathrm{~min}$ ) may result from a cooling process of very hot plasmas which increases the density of $\mathrm{Fe}^{+18}$ ions via recombinations. In some cases (e.g. 4[A, B, C], 11B, and 19[A, B]), intensity profiles show clearly two peaks, with the first one concurring with the initial Doppler shift pulse, supporting this assumption.

We find that most SUMER oscillation events happened without associated GOES flares, while all TRACE events were triggered by strong flares or filament destabilizations (about $70 \%$ were associated with $\mathrm{M}$ or $\mathrm{X}$ class flares) (Schrijver et al. 2002). This may suggest that the SUMER and TRACE loop oscillations are excited in different ways. The kink-mode oscillations of TRACE loops are most likely excited when a loop is hit by nearby erupting filaments and coronal mass ejections (Schrijver et al. 2002; Aschwanden et al. 2002). In contrast, the slow-mode waves in hot loops seen by SUMER could be excited by pressure disturbances associated with the injection of hot plasma at the oscillating loop's footpoint. Evidence for this exciter is provided by the footpoint brightening seen in SXT images of the recurring events on 29 September 2000 (see Sect. 3.3).
In this example, the manifestation of initial strong shifts of the same sign for all events implies a hot plasma outflow possibly accompanied with a disturbance along a large loop originating from one footpoint, supported also by the 3-D geometry, the associated SXR brightening and upwards moving EUV emission at this footpoint. The disturbance could be a strong impulsively generated pressure disturbance, which propagates as a slow mode magnetosonic wave along the loop and gets reflected at the opposite side (Nakariakov et al. 2000) to finally form standing waves. The associated hot plasma flow may contribute to strong emission of flare lines seen in SUMER. We speculate that the SXR brightening and inferred gas-pressure disturbance and plasma injection near the footpoint could be due to a sudden energy release caused by interactions between the large loop and a small twisted flux system. The magnetic reconnection in this configuration has been modeled (e.g., Yokoyama \& Shibata 1995; Démoulin et al. 1997; Karpen et al. 1998), which produces small "confined flares" (e.g., X-ray jets) with no substantial change of the magnetic structure (and in particular no opening of the closed field system), in agreement with the fact that the SUMER recurring events have similar features and with the absence of associated CMEs. Reconnection near the chromosphere also produces cool mass ejections (e.g., cool jets in $\mathrm{H}_{\alpha}$ or EUV) (Yokoyama \& Shibata 1995; Canfield et al. 1996). This can explain the upwards-moving EUV emission along the loop near the brightening footpoint. But the cool material could be too dense to reach the height where the SUMER slit is located, so explaining the absence of emission in cool lines. The reconnection between two loops located side-by-side can explain the existence of two components oscillating in antiphase, as seen in another example on 9 March 2001 (see also Wang et al. 2002a). Kliem et al. (2002) have also found indications for loop-loop interaction near one footpoint of a loop, causing the energy release and oscillations seen by SUMER. Similar to the case on 29 September 2000, Harrison (1987) find that the long-period $\mathrm{X}$-ray brightness pulsations also occur in such a configuration consisting of a large loop and a small loop with a common footpoint, consistent with the trigger mechanism we propose for the SUMER oscillations. Note that more than half of the oscillation events belong to recurring events, manifesting identical periods and initial shifts of the same sign (and not associated with CMEs), so that the involved magnetic structure and triggering mechanism may be similar to those discussed above.

The loop oscillation cases detected by TRACE are relatively rare (in $6 \%$ of 255 flares inspected), and this rarity is consistent with the proposal that transverse oscillations are preferentially found on loops at or near separatrices (Schrijver et al. 2002). In contrast, SUMER hot loop oscillations happen more frequently. They often recur $2-3$ times within a couple of hours. For all inspected data in 1999-2002, we identify 15 flares (1 X-class, 3 M-class and $11 \mathrm{C}$-class) detected by SUMER ( 9 in Fe XIX and 6 in Fe XXI). Of these 15 flares $6(40 \%)$ are associated with Doppler oscillations (see Table 2). For the 27 oscillation events, the peak intensity covers a large range on orders of $0.001-2 \mathrm{~W} \mathrm{~m}^{-2} \mathrm{sr}^{-1}$. Except 7 events excited by the 6 flares (their peak intensities lie in the range $0.02-2 \mathrm{~W} \mathrm{~m}^{-2} \mathrm{sr}^{-1}$ ) listed in Table 2, all the 
others are small events, with no associated GOES flares. Actually, there are a large number of small flux enhancements with peak intensity below $0.01 \mathrm{~W} \mathrm{~m}^{-2} \mathrm{sr}^{-1}$, showing weak shift fluctuations, which we did not include in our statistics. We also neglected many cases with only red-blue (or blue-red) shift alternation, or with non-periodic oscillations, which may also result from the same physical mechanism. These small events seen in hot flare lines with a high rate of occurrence may be correlated with microflares detected in $3.5-5.5 \mathrm{keV}$ X-ray emission characteristic of $T \sim 10$ MK (e.g., Porter et al. 1995). Therefore, future coordinated observations between SUMER and RHESSI in the SXR channels may help to identify the trigger of hot loop oscillations.

In addition, outward propagating longitudinal oscillations (slow waves) are detected in EUV radiation from the footpoints of large diffuse coronal loops by EIT and TRACE (De Moortel et al. 2002a,b). The disturbances travel with a propagation speed of the order of $122 \mathrm{~km} \mathrm{~s}^{-1}$ and periods of the order of $5 \mathrm{~min}$. These intensity oscillations can be continuously present for several hours, so that their triggers are completely different from the SUMER hot loop oscillations which are excited impulsively. De Moortel et al. (2002c) suggest that these intensity oscillations may be driven by the 3 min sunspot oscillations and the global $5 \mathrm{~min}$ solar oscillations.

\subsection{Propagation}

In some cases, we find Doppler oscillations exhibiting phase propagations along the slit with speeds of $8-102 \mathrm{~km} \mathrm{~s}^{-1}$. This feature seemingly does not agree with the property of standing waves. In comparison, the longitudinal oscillations detected in coronal loops in TRACE $171 \AA$ have propagating speeds in the range $70-235 \mathrm{~km} \mathrm{~s}^{-1}$, with a mean of $122 \pm 43 \mathrm{~km} \mathrm{~s}^{-1}$, in agreement with the sound speed $\left(c_{\mathrm{s}}=150 \mathrm{~km} \mathrm{~s}^{-1}\right)$ in a coronal plasma of $T=1 \mathrm{MK}$ (De Moortel et al. 2002a). Hence, these oscillations are interpreted as propagating slow mode waves. Here - because the SUMER oscillations occur in hot coronal loops, mainly detected in Fe XIX with a line formation temperature of $T=6.3 \mathrm{MK}$ - we estimate the propagation speed of slow waves to be $V \simeq c_{\mathrm{s}}=380 \mathrm{~km} \mathrm{~s}^{-1}$ if the ion temperature is assumed equal to the electron temperature. We find that this speed is much larger than the phase propagation speeds measured in Doppler oscillations. Considering that the magnetic field of an oscillating loop at the slit position makes an angle to the slit direction, e.g. $\gamma \gtrsim 75^{\circ}$, the expected propagating speed along the slit will be $V_{y}=c_{\mathrm{s}} \cos \gamma \lesssim 100 \mathrm{~km} \mathrm{~s}^{-1}$, which is in agreement with the observation. Cases with no clear phase propagation may be explained by loops with a small $\gamma$ angle. For example, for a case with $\gamma=45^{\circ}$, the transit time of slow waves through a typical extending scale $(\Delta Y=35 \mathrm{Mm})$ of the oscillations along the slit, will be $\Delta t \simeq \Delta Y / V_{y}=130 \mathrm{~s}$. Over this time, less than 3 Fe XIX images can be obtained with a cadence of $50 \mathrm{~s}$, thus it is not easy to discern the phase propagation feature in such a case.

Based on the above discussion, we cannot rule out the possibility that propagating waves are the cause of some of the observed oscillations. Also, the typically small number of oscillation periods suggests that propagation effects may often be visible. On the other hand, case $18 \mathrm{C}$ shows not only a clear phase propagation in Doppler shift, but also a 1/4-period phase relationship between Doppler velocity and intensity. The latter feature provides convincing evidence for slow standing waves. This wave mode also yields good agreement with the observed damping rates and their scalings in general (Ofman \& Wang 2002). Therefore, we suggest that the origin of this phase propagation could be related to the fine structure of coronal loops and the trigger mechanism of oscillation events on the basis of the following discussion.

For most of the observations, the SUMER slit was placed at a height $H \simeq 100^{\prime \prime}$ above the limb. Consider a semicircular coronal loop located in a vertical plane with its top just at the slit (i.e. with a loop radius $R=H$ ). We then have a loop length $L \simeq 230 \mathrm{Mm}$, in agreement with the typical length $(\simeq 220 \mathrm{Mm})$ of coronal loops seen by TRACE (Aschwanden et al. 2002). Further, let the loop width be $w=$ $9 \mathrm{Mm}$ (Aschwanden et al. 2002) and its azimuth angle to be $\alpha=45^{\circ}$. From this we estimate the intersection length of the loop top with the slit as $\Delta Y \simeq 2 \sin \alpha \sqrt{(R+w / 2)^{2}-R^{2}}=$ $37 \mathrm{Mm}$, in good agreement with the scale $(35 \pm 21 \mathrm{Mm})$ of the observed oscillatory regions along the slit (Table 4). When the loop has $\alpha \simeq 0^{\circ}$ (i.e., the loop is directed nearly along the westeast direction), $\Delta Y \simeq w=9 \mathrm{Mm}$, close to the lower limit of the observed $\Delta Y$. These estimates indicate that the oscillatory Doppler shifts observed in a region can be caused by a single coronal loop. In this case the differences of intensity and line width along the slit in an oscillatory region may be due to the fine structure of a coronal loop which may consist of a large number of fine threads as seen in TRACE 171 and $195 \AA$ images (Aschwanden et al. 2000); different threads can have different densities and possibly different temperatures. We cannot, however, exclude the possibility that these differences may be due to several neighboring parallel loops with a small azimuth angle relative to the E-W direction. If magnetic reconnection triggers thermal energy release at a loop's footpoint in a certain thread, the produced gas-pressure disturbance will affect the other threads at a slightly later time than the directly involved thread, thus exciting slow waves in those threads with phases delayed relative to the slow wave in the thread directly related to the trigger. Recall that the phase delay seems to propagate along the slit from the strong emission region to the faint ones, supporting this idea.

\section{Conclusions}

SUMER spectral observations have revealed a new kind of damped oscillation in hot coronal loops. In six selected examples, we analyzed in detail the features of time series of Doppler shift, intensity and line width, and explored the trigger of oscillation events and oscillating loops using SXT images. We carried out quantitative measurements of physical parameters of 54 oscillations in 27 brightening events seen in flare lines. With this study we have thus been able to obtain an overview of the properties of the loop oscillations recorded by SUMER. We compared the statistical results of the periods, decay times, and amplitudes with TRACE results. We have also 
discussed the wave mode and damping mechanism of hot loop oscillations. Based on these analyses, we come to the following conclusions.

Doppler shift oscillations were detected only in flare lines like Fe XIX and Fe XXI of $T>6 \mathrm{MK}$, consistent with their association with hot SXR loops. For all cases, the oscillations are excited impulsively, as evidenced by the presence of an initial large Doppler shift pulse and impulsive profiles of intensity and line width. Because oscillation events have a relatively high frequency of occurrence (e.g. often showing a recurring behaviour) and only a small number of them are associated with GOES flares, they may be triggered by micro- or subflares. The recurring events show initial shifts of the same sign, consistent with exciters (e.g., pressure disturbance and injected hot plasma) of loop oscillations coming from one footpoint. This interpretation is supported by a case study which includes a comparison with SXT data. Phase propagation of the oscillations, together with variations of intensity and line width along the slit, can be explained by the excitation of the oscillation at a footpoint of an inhomogeneous coronal loop, e.g. a loop with fine (or multi-thread) structure.

The SUMER oscillations have distinctly longer periods than the TRACE transverse oscillations, but a relatively similar decay rate. Various lines of evidence indicating that they are compressive slow magnetoacoustic standing waves are presented, thus supporting the conclusions reached by Wang et al. (2003). For example, the phase speeds inferred from the oscillation period and loop length approximately match the sound speeds in hot coronal loops. Furthermore, to make the period of the global kink mode match the observed period, requires an unusual coronal loop environment of plasma $\beta \geq 1$. A large thermal conduction due to the high temperature of hot loops can explain the observed rapid damping on a timescale of the order of a wave period (Ofman \& Wang 2002). The scaling of the dissipation time of slow mode waves with period is in good agreement with the scaling for 49 oscillation cases.

There are still some issues far from clearly understood, such as the excitation mechanism of slow mode waves, the buildup of standing waves, the coronal loop environment required for quick damping $\left(\tau_{\mathrm{d}} / P \simeq 1-2\right)$, reasons for the absence of intensity fluctuations in many cases, the true interpretation of the occasionally seen phase propagation along the slit. These issues need further studies both in observation and theory in the future.

Acknowledgements. SUMER is financially supported by DLR, CNES, NASA and the ESA PRODEX programme. SOHO is a project of international co-operation between ESA and NASA. Yohkoh is a mission of the Institute of Space and Astronautical Science (Japan), with participation from the US and UK.

\section{References}

Aschwanden, M. J. 1987, Sol. Phys., 111, 113

Aschwanden, M. J. 2002, NATO Advanced Workshop: Turbulence, Waves and Instabilities, Budapest, in press

Aschwanden, M. J., Newmark, J. S., Delaboudiniere, J. P., et al. 1999a, ApJ, 515, 842
Aschwanden, M. J., Fletcher, L., Schrijver, C. J., \& Alexander, D. 1999b, ApJ, 520, 880

Aschwanden, M. J., Nightingale, R. W., \& Alexander, D. 2000, ApJ, 541,1059

Aschwanden, M. J., Pontieu, B. D., Schrijver, C. J., \& Title, A. 2002, Sol. Phys., 206, 99

Berghmans, D., \& Clette, F. 1999, Sol. Phys., 186, 207

Canfield, R. C., Reardon, K. P., Leka, K. D., et al. 1996, ApJ, 464, 1016

De Moortel, I., Ireland, J., \& Walsh, R. W. 2000, A\&A, 355, L23

De Moortel, I., Ireland, J., Walsh, R. W., \& Hood, A. W. 2002a, Sol. Phys., 209, 61

De Moortel, I., Hood, A. W., Ireland, J., \& Walsh, R. W. 2002b, Sol. Phys., 209, 89

De Moortel, I., Ireland, J., Hood, A. W., \& Walsh, R. W. 2002c, A\&A, 387, L13

Démoulin, P, Bagalá, L. G., Mandrini, C. H., et al. 1997, A\&A, 325, 305

Gary, G. A. 2001, Sol. Phys., 203, 71

Harrison, R. A. 1987, A\&A, 182, 337

Karpen, J. T., Antiochos, S. K., DeVore, C. R., \& Golub, L. 1998, ApJ, 495, 491

Kliem, B., Dammasch, I. E., Curdt, W., \& Wilhelm, K. 2002, ApJ, 568, L61

McKenzie, D. E., \& Mullan, D. J. 1997, Sol. Phys., 176, 127

Nakariakov, V. M., Ofman, L., DeLuca, E. E., et al. 1999, Science, 285,862

Nakariakov, V. M., Verwichte, E., Berghmans, D., \& Robbrecht, E. 2000, A\&A, 362, 1151

Nakariakov, V. M., \& Ofman, L. 2001, A\&A, 372, L53

Ofman, L. 2002, ApJ, 568, L135

Ofman, L., \& Aschwanden, M. J. 2002, ApJ, 576, L153

Ofman, L., \& Wang, T. J. 2002, ApJ, 580, L85

Porter, J. G., Fontenla, J. M., \& Simnett, G. M. 1995, ApJ, 438, 472

Robbrecht, E., Verwichte, E., Hochedez, J. F., et al. 2001, A\&A, 370, 591

Roberts, B., Edwin, P. M., \& Benz, A. O. 1984, ApJ, 279, 857

Roberts, B. 2000, Sol. Phys., 193, 139

Sakurai, T., Ichimoto, K., Raju, K. P., \& Singh, J. 2002, Sol. Phys., 209, 265

Schmelz, J. T., Holman, G. D., Brosius, J. W., \& Willson, R. F. 1994, ApJ, 434, 786

Schrijver, C. J., \& Brown, D. S. 2000, ApJ, 537, L69

Schrijver, C. J., Aschwanden, M. J., \& Title, A. M. 2002, Sol. Phys., 206, 69

Švestka, Z., Dennis, B. R., Pick, M., et al. 1982, Sol. Phys., 80, 143

Švestka, Z. 1994, Sol. Phys., 152, 505

Tsiklauri, D., \& Nakariakov, V. M. 2001, A\&A, 379, 1106

Tsuneta, S., Acton, L., Bruner, M., et al. 1991, Sol. Phys., 136, 37

Wang, T. J., Solanki, S. K., Curdt, W., Innes, D. E., \& Dammasch, I. E. 2002a, ApJ, 574, L101

Wang, T. J., Solanki, S. K., Curdt, W., Innes, D. E., \& Dammasch, I. E. 2002b, in Proc. 11th SOHO Workshop: From Solar Minimum to Maximum, ed. A. Wilson (ESA SP-508, Noordwijk: ESA), 465

Wang, T. J., Solanki, S. K., Curdt, W., Innes, D. E., \& Dammasch, I. E. 2002c, in Proc. IAU Colloq. 188: Magnetic Coupling of the Solar Atmosphere, ed. H. Sawaya-Lacoste (ESA SP-505, Noordwijk: ESA), 199

Wang, T. J., Solanki, S. K., Innes, D. E., Curdt, W., \& Marsch, E. 2003, A\&A, 402, L17

Wilhelm, K., Curdt, W., Marsch, E., et al. 1995, Sol. Phys., 162, 189

Yokoyama, T., \& Shibata, K. 1995, Nature, 375, 42 
T. J. Wang et al.: Hot coronal loop oscillations observed by SUMER, Online Material $p 1$

\section{Online Material}


T. J. Wang et al.: Hot coronal loop oscillations observed by SUMER, Online Material $p 2$

Table 1. Time series analysis of Doppler shift oscillations ${ }^{\mathrm{a}}$.

\begin{tabular}{|c|c|c|c|c|c|c|c|c|c|c|c|c|}
\hline $\begin{array}{l}\text { Osci. } \\
\text { Comp. }\end{array}$ & $\begin{array}{c}t_{0} \\
\text { (UT) }\end{array}$ & $\begin{array}{r}V_{\mathrm{D}} \\
\left(\mathrm{km} \mathrm{s}^{-1}\right)\end{array}$ & $\begin{array}{r}V_{\mathrm{m}} \\
\left(\mathrm{km} \mathrm{s}^{-1}\right)\end{array}$ & $\begin{array}{r}P \\
(\min )\end{array}$ & $\begin{array}{r}\phi \\
(\mathrm{rad})\end{array}$ & $\begin{array}{r}\tau_{\mathrm{d}} \\
(\min )\end{array}$ & $\begin{array}{r}A \\
(\mathrm{Mm})\end{array}$ & $N_{\mathrm{P}}$ & $\begin{array}{l}\Delta T_{\mathrm{IV}} \\
(\mathrm{min})\end{array}$ & $\begin{array}{r}\Delta T_{\mathrm{WV}} \\
(\mathrm{min})\end{array}$ & $\begin{array}{r}\Delta T_{\mathrm{I}} \\
(\mathrm{min})\end{array}$ & $N_{\mathrm{I}}$ \\
\hline 1A*, Fig. 4 & 06:33:56 06-Nov.-99 & 110 & 62 & 11.7 & -0.01 & 8.8 & 6.7 & 2.5 & 9.0 & 4.0 & 20 & 1 \\
\hline 2A, Fig. 5 & 02:20:09 29-Sep.-00 & 89 & 74 & 29.1 & 0.03 & 22.0 & 20.2 & 2 & 5.4 & 0 & 16 & 1 \\
\hline $2 \mathrm{~B}$ & 02:20:09 29-Sep.-00 & 78 & 53 & 30.0 & -0.01 & 28.9 & 14.8 & 2 & 2.7 & 0 & 38 & 3 \\
\hline $3 \mathrm{C}$ & 03:39:13 29-Sep.-00 & 64 & 51 & 25.7 & -0.01 & 21.2 & 12.4 & 2 & 2.7 & 0 & 15 & 1 \\
\hline $3 \mathrm{D}$ & 03:39:13 29-Sep.-00 & 37 & 43 & 29.6 & 0.70 & 28.0 & 11.9 & 2 & 0 & -2.7 & 19 & 1 \\
\hline 4A, Fig. 7 & 13:12:14 29-Sep.-00 & 191 & 144 & 25.1 & 2.25 & 12.5 & 32.8 & 2 & 2.7 & 5.4 & 81 & 2 \\
\hline 4B & 13:06:49 29-Sep.-00 & 192 & 121 & 25.7 & -0.16 & 12.6 & 28.2 & 2 & 0 & 0 & 141 & 3 \\
\hline $4 \mathrm{C}$ & 13:12:14 29-Sep.-00 & 189 & 107 & 31.1 & 2.55 & 18.7 & 30.7 & 2 & 16.4 & 0 & 55 & 3 \\
\hline 5A, Fig. 2 & 15:57:45 09-Mar.-01 & 44 & 67 & 17.9 & 3.07 & 13.7 & 11.2 & 2 & 5.6 & -2.8 & 27 & 1 \\
\hline $5 \mathrm{~B}$ & 15:57:45 09-Mar.-01 & 14 & 12 & 15.7 & -0.92 & 11.9 & 1.7 & 2 & 0 & 0 & 23 & 2 \\
\hline $6 \mathrm{C}$ & 17:31:07 09-Mar.-01 & 70 & 48 & 14.2 & -0.17 & 15.0 & 6.4 & 3 & 8.5 & -2.8 & 31 & 1 \\
\hline $6 \mathrm{D}$ & 17:31:07 09-Mar.-01 & 30 & 48 & 15.7 & 2.83 & 19.0 & 7.2 & 3 & 11.3 & 2.8 & 17 & 2 \\
\hline 7A, Fig. B.1 & 00:36:18 11-Apr.-02 & 108 & 136 & 14.4 & 3.09 & 12.3 & 18.3 & 3 & 0 & -0.8 & 14 & 1 \\
\hline $8 \mathrm{~B}$ & 01:33:29 11-Apr.-02 & 28 & 28 & 11.4 & -2.93 & 10.1 & 3.0 & 2 & 5 & 9.1 & 35 & 2 \\
\hline $8 \mathrm{C}$ & 01:33:29 11-Apr.-02 & 31 & 27 & 13.5 & 0.23 & 10.8 & 3.4 & 2 & 2.5 & 0 & 20 & 1 \\
\hline 9A, Fig. B.2 & 07:48:45 11-Apr.-02 & 55 & 35 & 10.8 & -0.20 & 6.7 & 3.5 & 3 & -1.7 & -4.1 & 26 & 2 \\
\hline $9 \mathrm{~B}$ & 07:47:55 11-Apr.-02 & 49 & 29 & 11.0 & -0.35 & 6.1 & 2.9 & 3 & -0.8 & -2.5 & 24 & 2 \\
\hline $9 \mathrm{C}$ & 07:47:55 11-Apr.-02 & 67 & 49 & 11.9 & -0.12 & 7.5 & 5.4 & 1.5 & 0.4 & -0.4 & 12 & 1 \\
\hline 10A, Fig. 9 & 19:20:51 11-Apr.-02 & 117 & 116 & 14.8 & 2.80 & 12.8 & 16.1 & 2 & 0.8 & -0.8 & 16 & 2 \\
\hline $11 \mathrm{~B}$ & 20:20:30 11-Apr.-02 & 295 & 183 & 17.2 & -0.52 & 9.0 & 28.7 & 2 & 11.6 & -0.8 & 24 & 2 \\
\hline $11 \mathrm{C}$ & 20:20:30 11-Apr.-02 & 100 & 76 & 13.1 & -0.22 & 9.2 & 9.3 & 1.5 & 3.3 & -0.8 & 27 & 1 \\
\hline $11 \mathrm{D}$ & 20:20:30 11-Apr.-02 & 44 & 30 & 17.1 & -0.49 & 17.2 & 4.9 & 2 & 2.5 & -0.8 & 21 & 1 \\
\hline $12 \mathrm{E}$ & 21:19:19 11-Apr.-02 & 45 & 39 & 17.6 & 0.41 & 16.4 & 6.5 & 1.5 & -0.6 & -0.8 & 10 & 1 \\
\hline 13A, Fig. B.3 & 05:08:26 12-Apr.-02 & 38 & 32 & 19.1 & 2.69 & 28.0 & 5.8 & 2 & 0.8 & 5.1 & 23 & 1 \\
\hline $13 \mathrm{~B}$ & 05:08:26 12-Apr.-02 & 36 & 26 & 22.7 & 2.52 & 17.7 & 5.5 & 2 & 2.5 & 4.1 & 25 & 1 \\
\hline $14 \mathrm{C}$ & 07:42:39 12-Apr.-02 & 38 & 38 & 20.0 & 3.11 & 14.9 & 7.1 & 1.5 & 2.5 & 0.8 & 11 & 1 \\
\hline 14D & 07:42:39 12-Apr.-02 & 29 & 38 & 25.0 & -2.02 & 25.4 & 9.1 & 2 & 0.8 & -1.7 & 21 & 1 \\
\hline 15A, Fig. 10 & 10:18:03 12-Apr.-02 & 190 & 116 & 18.1 & -0.11 & 11.7 & 19.5 & 2 & 3.4 & 2.5 & 41 & 1 \\
\hline 15B & 10:11:27 12-Apr.-02 & 134 & 71 & 19.4 & -0.23 & 13.2 & 12.8 & 2 & 14.3 & 1.7 & 55 & 1 \\
\hline $15 \mathrm{C}$ & 10:11:27 12-Apr.-02 & 229 & 124 & 19.2 & -0.31 & 9.9 & 21.7 & 3 & 10.7 & 5.8 & 46 & 1 \\
\hline $15 \mathrm{D}$ & 10:11:27 12-Apr.-02 & 87 & 72 & 16.0 & -1.67 & 18.7 & 10.9 & 3 & 4.1 & -0.8 & 62 & 1 \\
\hline $16 \mathrm{E}$ & 11.35:17 12-Apr.-02 & 64 & 52 & 20.0 & 0.00 & 16.0 & 9.7 & 1.5 & 0.8 & -1.7 & 13 & 1 \\
\hline $16 \mathrm{~F}$ & 11:31:10 12-Apr.-02 & 101 & 74 & 16.0 & 0.16 & 10.1 & 11.0 & 2 & 1.7 & 0 & 25 & 1 \\
\hline 17A, Fig. 11 & 23:26:52 15-Apr.-02 & 75 & 52 & 16.4 & -0.46 & 12.6 & 7.9 & 2 & 52.2 & -2.5 & 116.2 & 2 \\
\hline $18 \mathrm{~B}$ & 01:05:34 16-Apr.-02 & 14 & 11 & 16.1 & -0.18 & 22.1 & 1.7 & 3 & 5.8 & 6.6 & 38.2 & 1 \\
\hline $18 \mathrm{C}$ & 00:47:25 16-Apr.-02 & 18 & 16 & 17.6 & 3.22 & 36.8 & 2.6 & 5 & -2.5 & -0.8 & 50.6 & 3 \\
\hline $18 \mathrm{D}$ & 01:03:05 16-Apr.-02 & 14 & 14 & 18.1 & -3.24 & 32.6 & 2.4 & 4 & 1.7 & 1.7 & 42.3 & 1 \\
\hline 19A, Fig. B.4 & 06:25:13 16-Apr.-02 & 319 & 226 & 16.4 & -0.14 & 7.8 & 33.6 & 1.5 & 0 & 1.7 & 12 & 2 \\
\hline 19B & 06:25:13 16-Apr.-02 & 275 & 165 & 13.4 & 0.09 & 5.7 & 19.8 & 2 & 0 & -1.8 & 18 & 2 \\
\hline 20A, Fig. B.5 & 11:18:10 17-Apr.-02 & 157 & 129 & 13.2 & 0.08 & 8.4 & 15.8 & 2.5 & 21.5 & -2.5 & 40 & 2 \\
\hline $20 \mathrm{~B}$ & 11:24:46 17-Apr.-02 & 59 & 41 & 15.4 & -3.12 & 12.0 & 5.9 & 2.5 & 5.8 & -1.7 & 37 & 2 \\
\hline $20 \mathrm{C}$ & 11:20:39 17-Apr.-02 & 105 & 101 & 13.1 & 2.42 & 9.7 & 12.3 & 3 & 16.5 & 3.3 & 26 & 2 \\
\hline 21A, Fig. B.6 & 11:04:37 19-Apr.-02 & 127 & 116 & 18.0 & -1.95 & 9.7 & 19.1 & 2 & 3.3 & 0.8 & 10 & 1 \\
\hline 21B & 11:04:37 19-Apr.-02 & 241 & 234 & 21.6 & -1.42 & 7.3 & 43.7 & 2 & 0.0 & 3.5 & 21 & 2 \\
\hline 22A*, Fig. B.7 & 18:28:01 23-Apr.-02 & 158 & 59 & 21.1 & -3.14 & 14.5 & 11.6 & 2 & 1.7 & 5.8 & 17 & 1 \\
\hline 23A, Fig. B.8 & 16:18:58 24-Apr.-02 & 62 & 46 & 13.9 & -3.25 & 12.4 & 6.0 & 2.5 & 5.0 & 0.8 & 20 & 1 \\
\hline 24A, Fig. B.9 & 15:50:27 26-Apr.-02 & 69 & 62 & 13.9 & 0.54 & 15.2 & 8.2 & 2 & 5.8 & 4.1 & 20 & 1 \\
\hline 25A, Fig. B. 10 & 14:49:39 08-May-02 & 209 & 189 & 23.9 & 0.84 & 7.9 & 38.9 & 2 & 20.5 & 8.5 & 48 & 1 \\
\hline $26 \mathrm{~B}$ & 15:47:47 08-May-02 & 67 & 91 & 17.7 & -0.05 & 13.4 & 15.0 & 2.5 & 14.1 & 0.0 & 46 & 1 \\
\hline $26 \mathrm{C}$ & 15:45:39 08-Мау-02 & 90 & 72 & 15.1 & -0.21 & 8.8 & 10.1 & 2.5 & 24.8 & 1.1 & 46 & 1 \\
\hline $26 \mathrm{D}$ & 15:45:39 08-May-02 & 59 & 59 & 18.2 & -0.1 & 22.5 & 10.3 & 3 & 8.8 & -3.2 & 55 & 3 \\
\hline 27A*, Fig. B.11 & 08:08:26 15-May-02 & 62 & 35 & 10.0 & -0.18 & 12.1 & 3.4 & 3.5 & 42.5 & 5.0 & 96 & 3 \\
\hline $27 \mathrm{~B}^{*}$ & 08:08:26 15-May-02 & 84 & 87 & 7.1 & -0.11 & 6.5 & 5.8 & 3.5 & 52.5 & 3.8 & 85 & 3 \\
\hline $27 C^{*}$ & 08:13:26 15-Мay-02 & 19 & 19 & 10.2 & -1.32 & 16.8 & 1.8 & 2 & 50.0 & 1.3 & 76 & 1 \\
\hline
\end{tabular}

${ }^{\mathrm{a}} t_{0}$ is the start time of the modeled time series, $V_{\mathrm{D}}$ the Doppler velocity amplitude derived by the best fit of a damped sine-function to the oscillations, $V_{\mathrm{m}}$ the maximum Doppler velocity amplitude measured from the data, $P$ the oscillation period, $\phi$ the phase of the oscillation, $\tau_{\mathrm{d}}$ the decay time $\left(\tau_{\mathrm{d}}=1 / \lambda\right), A$ the displacement amplitude (defined as $\left.A=V_{\mathrm{m}} /\left(\omega^{2}+\lambda^{2}\right)^{1 / 2}\right), N_{\mathrm{P}}$ the number of periods over which an oscillation was detected, $\Delta T_{\mathrm{IV}}$ and $\Delta T_{\mathrm{WV}}$ the time lags of the maximum intensity peak and the maximum Doppler line width peak relative to the maximum Doppler-shift pulse, respectively, $\Delta T_{\mathrm{I}}$ the duration of intensity peak (defined as the time spent at brightness above $1 / e$ of the maximum), $N_{\mathrm{I}}$ the number of intensity peaks.

${ }^{\mathrm{b}}$ In the cases marked with asterisks, the Doppler oscillations were observed in the line Fe XXI; for the other cases the oscillations were observed in Fe XIX. 
T. J. Wang et al.: Hot coronal loop oscillations observed by SUMER, Online Material $p 3$

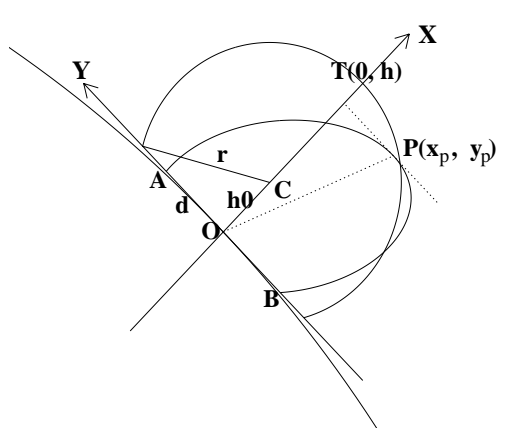

Fig. A.1. Projection of a hypothetical circular loop onto the solar limb. Cartesian coordinate system $O X Y Z$ is defined at the midpoint $(O)$ between the two footpoints $A$ and $B ; O X$ is an extension of the solar radius at $O, O Y$ is tangential to the limb towards the north. The loop plane has an inclination angle $\theta$ to the vertical plane passing through the loop's footpoints, which is rotated by an azimuthal angle $\beta$ to the NS vertical plane $(X O Y) . P\left(x_{\mathrm{p}}, y_{\mathrm{p}}\right)$ is the apex position of the loop.

\section{Appendix A: Derivation of geometrical parameters of coronal loops above the limb}

Let $O$ in Fig. A.1 be the midpoint of the line joining the footpoints $A$ and $B$ of a loop and $d$ be half of the distance between the footpoints in the plane $X O Y$. We define the loop geometry by a circle with radius $r$ and height $h_{0}$ of the circle's center above the solar surface. In the case that the plane of the loop is parallel to the plane $X O Y, C$ is the circle's center (with $O C$ defined as $h_{0}$ ) and $T$ is the circle's apex (with $O T$ defined as $h$ ). In the case that the loop plane has an inclination angle $\theta$ to the vertical and an azimuthal angle $\beta$ between the footpoint baseline and the north-south direction (counterclockwise), we define $P\left(x_{\mathrm{p}}, y_{\mathrm{p}}\right)$ as the position of the loop's apex. Typically the distance between the footpoints is small compared to the solar radius, so that we may neglect curvature and regard the loop as projecting upward from the plane through $O$ tangential to the solar surface. Using the coordinate transformation (e.g. formula (A5) in Aschwanden et al. 1999a), we obtain the equations,

$$
\begin{aligned}
x_{\mathrm{p}} & =h \cos \theta, \\
y_{\mathrm{p}} & =-h \sin \theta \sin \beta, \\
d & =r \sqrt{1-\left(h_{0} / r\right)^{2}} \cos \beta .
\end{aligned}
$$

Defining $h_{0} / r \equiv c$, and considering $h=h_{0}+r$, we obtain from Eq. (A.3),

$D=h \cos \beta$,

where the quantity $D$ is defined by $D=d \sqrt{1+c} / \sqrt{1-c}$. Solving Eqs. (A.1), (A.2) and (A.4) for $\theta, \beta$ and $h$, we obtain

$h=\left[\frac{1}{2}\left(x_{\mathrm{p}}^{2}+y_{\mathrm{p}}^{2}+D^{2}\right)+\frac{1}{2} \sqrt{\left(x_{\mathrm{p}}^{2}+y_{\mathrm{p}}^{2}+D^{2}\right)^{2}-4 x_{\mathrm{p}}^{2} D^{2}}\right]^{1 / 2}$

then,

$r=\frac{h}{1+c}$,

$$
\begin{aligned}
& \theta=\cos ^{-1}\left(\frac{x_{\mathrm{p}}}{h}\right), \\
& \beta=\cos ^{-1}\left(\frac{D}{h}\right) .
\end{aligned}
$$

If we assume that the observed loop has the midpoint between its footpoints exactly above the limb, we can determine the positions of footpoints $A$ and $B$ and hence measure their distance, $2 d$. Because the position coordinate, $x$, of the observed loop reaches the maximum, $x_{\mathrm{p}}$, at the apex when the loop has a circular shape, we can determine the apex position $P\left(x_{\mathrm{p}}, y_{\mathrm{p}}\right)$ of the loop. The ratio $c\left(=h_{0} / r\right)$ is a free parameter. Therefore, given a value of $c$, we can derive the geometrical parameters $(r, \theta$, and $\beta$ ) of the observed loop from the measurements of $x_{\mathrm{p}}, y_{\mathrm{p}}$, and $d$ using Eqs. (A.5)-(A.8). We obtain the value of free parameter $c$ by minimization of the difference between the observed loop image and the projected image of the modelled loop. Note that as it is difficult to discern which foot of the observed loop is closer to the observer, we cannot determine whether the loop plane is inclined towards or away from the observer. For example, in the case demonstrated in Fig. A.1, we deduce from the apex position of the projected loop (i.e. $\left.y_{\mathrm{p}}<0\right)$ that the loop plane inclines towards the observer if the $A$-foot is closer to the observer, while it inclines away from the observer if the $B$-foot is closer to the observer.

\section{Appendix B: Doppler oscillation cases}

Further examples of Doppler shift oscillations recorded by SUMER are shown in Figs. B.1-B.11. 
T. J. Wang et al.: Hot coronal loop oscillations observed by SUMER, Online Material $p 4$

(a) Line-of-sight Doppler shift
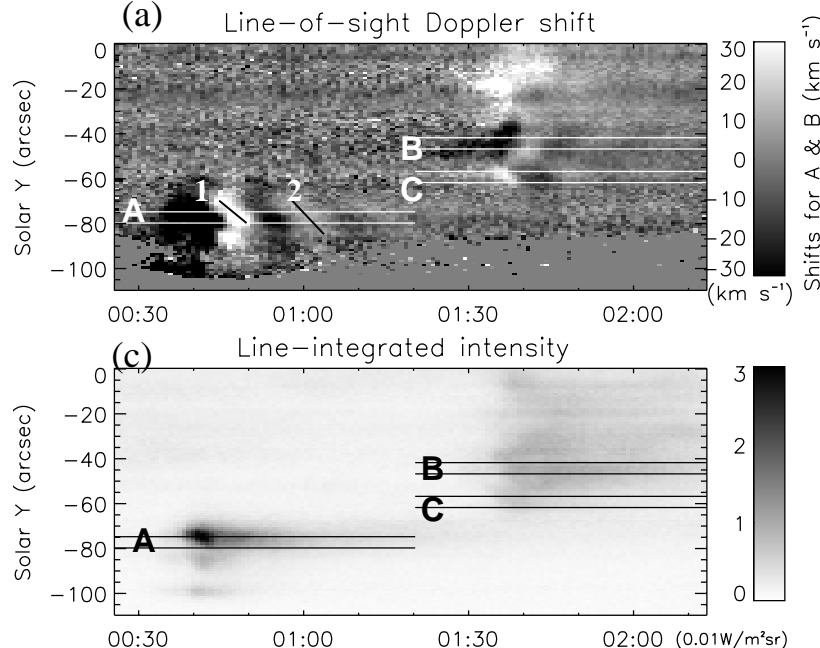

(e)

$01: 00 \quad 01: 30$
Line width
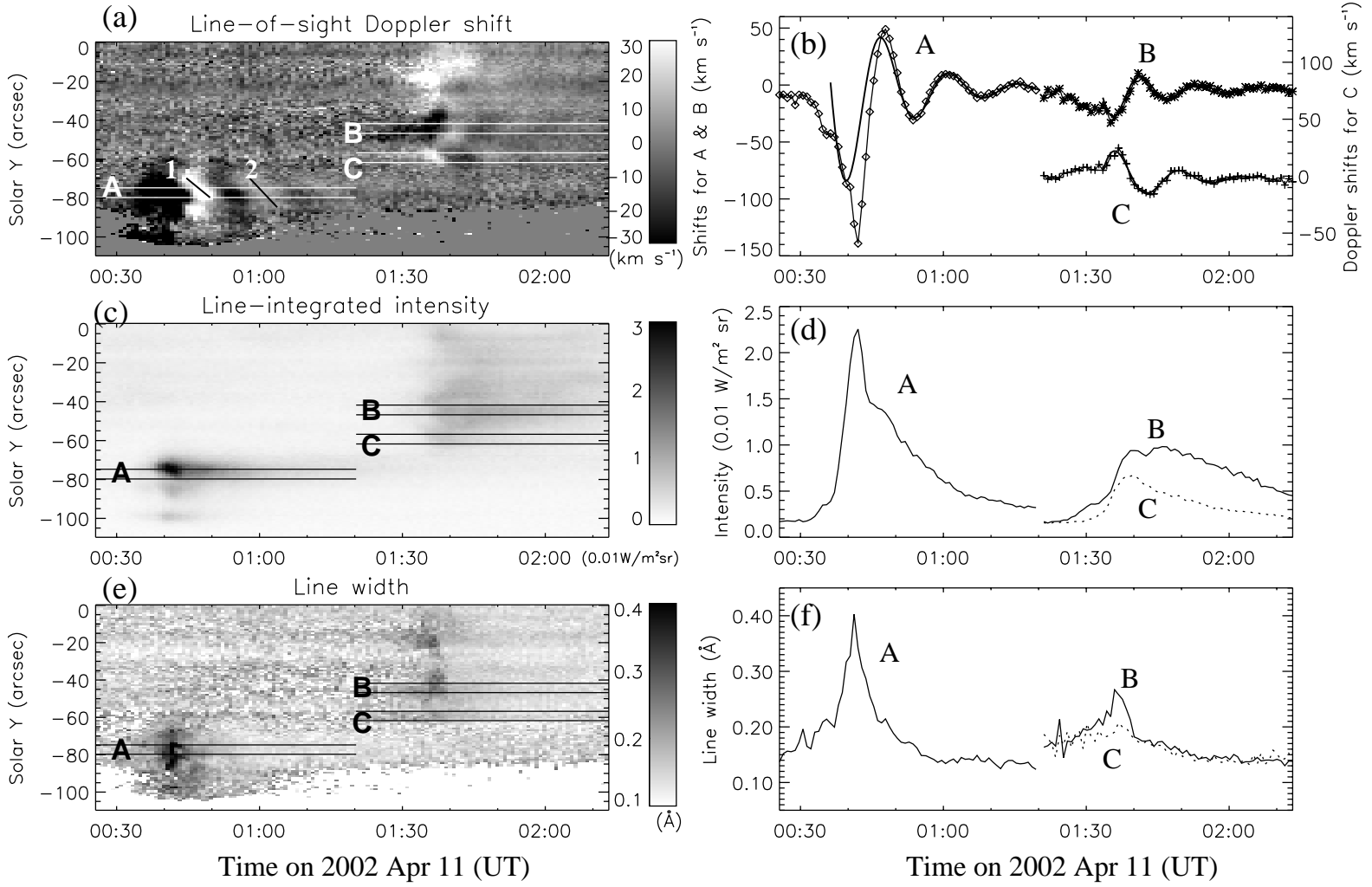

Fig. B.1. Doppler oscillation events (Nos. 7A, 8B, 8C) in the Fe XIX line on 11 April 2002.
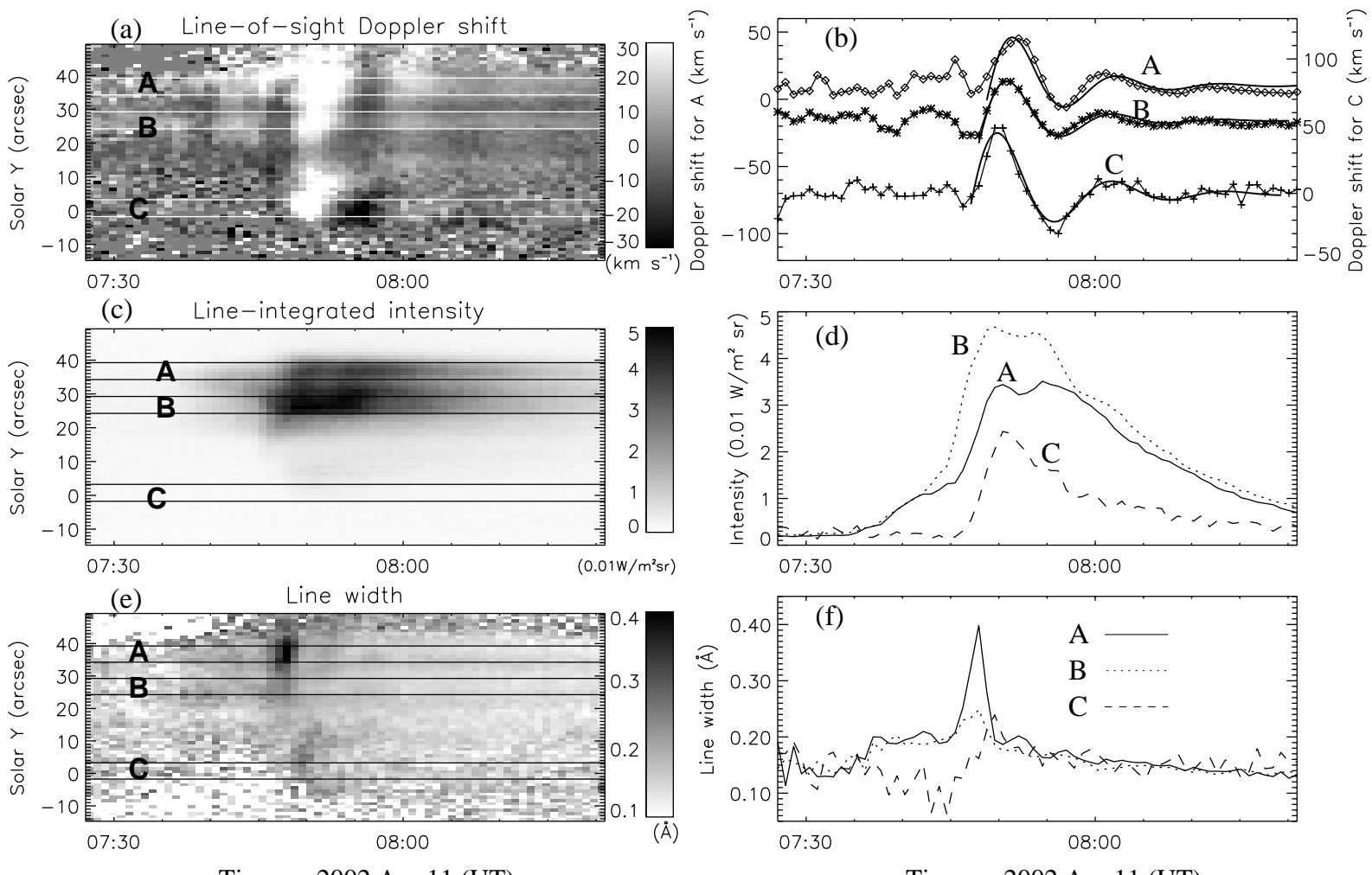

Time on 2002 Apr 11 (UT)

Time on 2002 Apr 11 (UT)

Fig. B.2. Doppler oscillation events (Nos. 9A, 9B, 9C) in the Fe XIX line on 11 April 2002. In b) the curve B is plotted relative to the left $y$-axis, but shifted by $-20 \mathrm{~km} \mathrm{~s}^{-1}$. 
T. J. Wang et al.: Hot coronal loop oscillations observed by SUMER, Online Material p 5
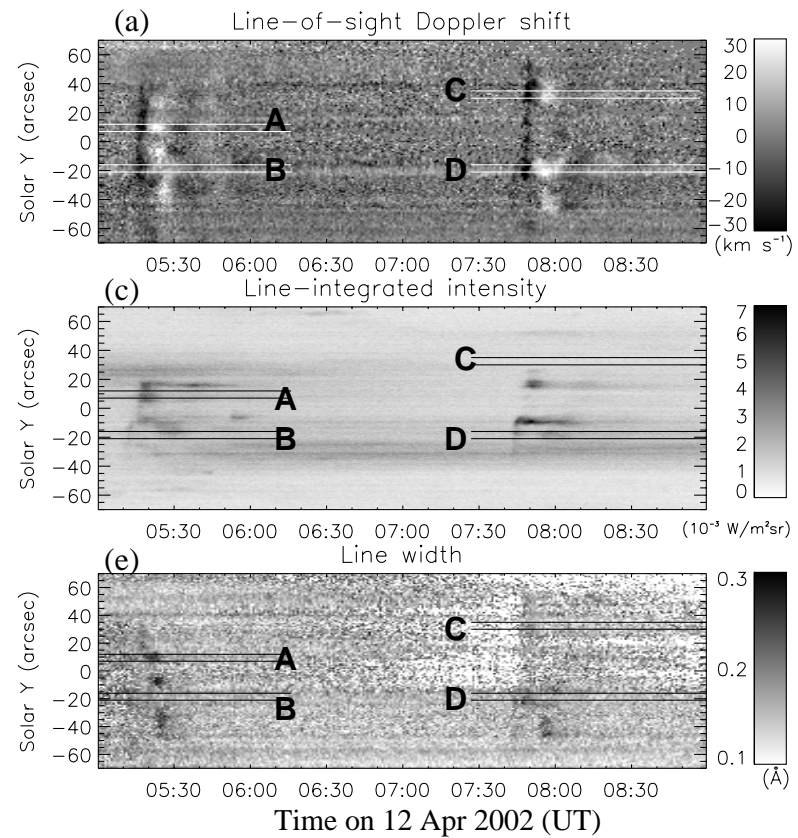

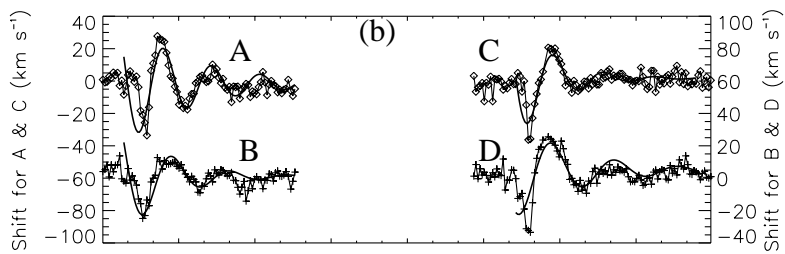

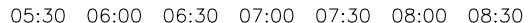
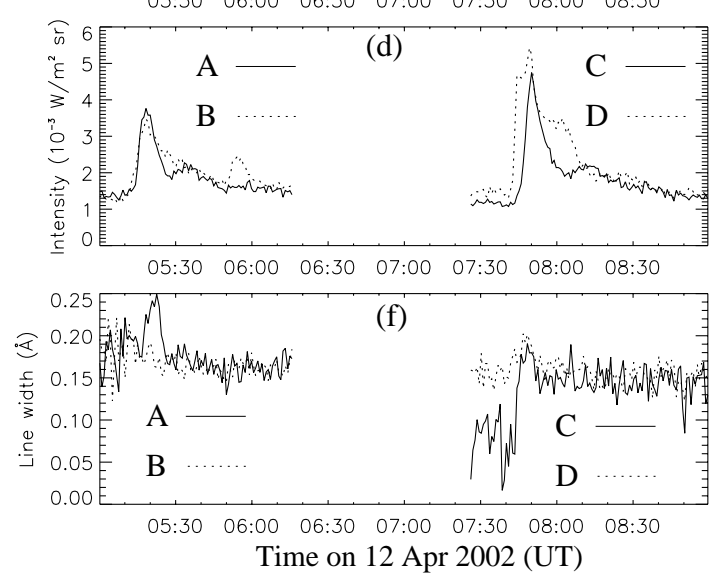

Fig. B.3. Doppler oscillation events (Nos. 13A, 13B, 14C, 14D) in the Fe XIX line on 12 April 2002.
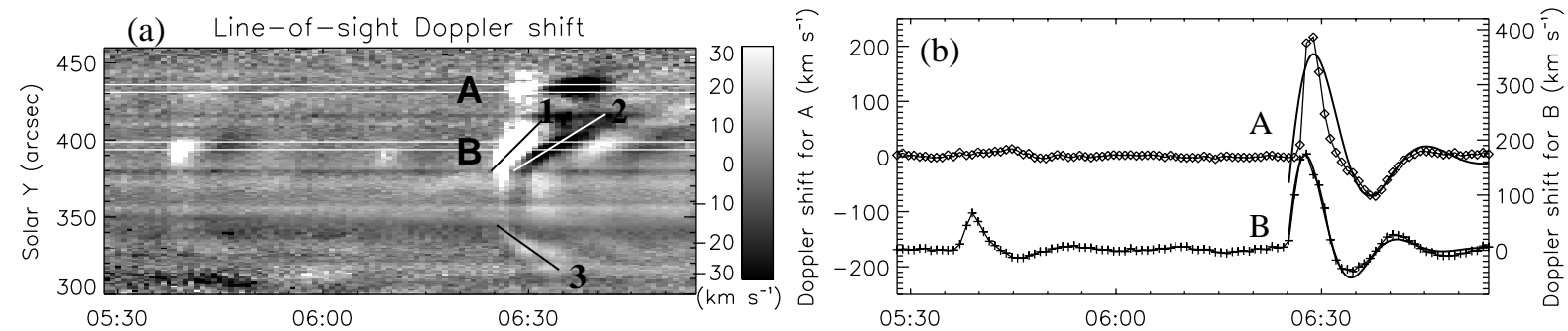

(c) Line-integrated intensity
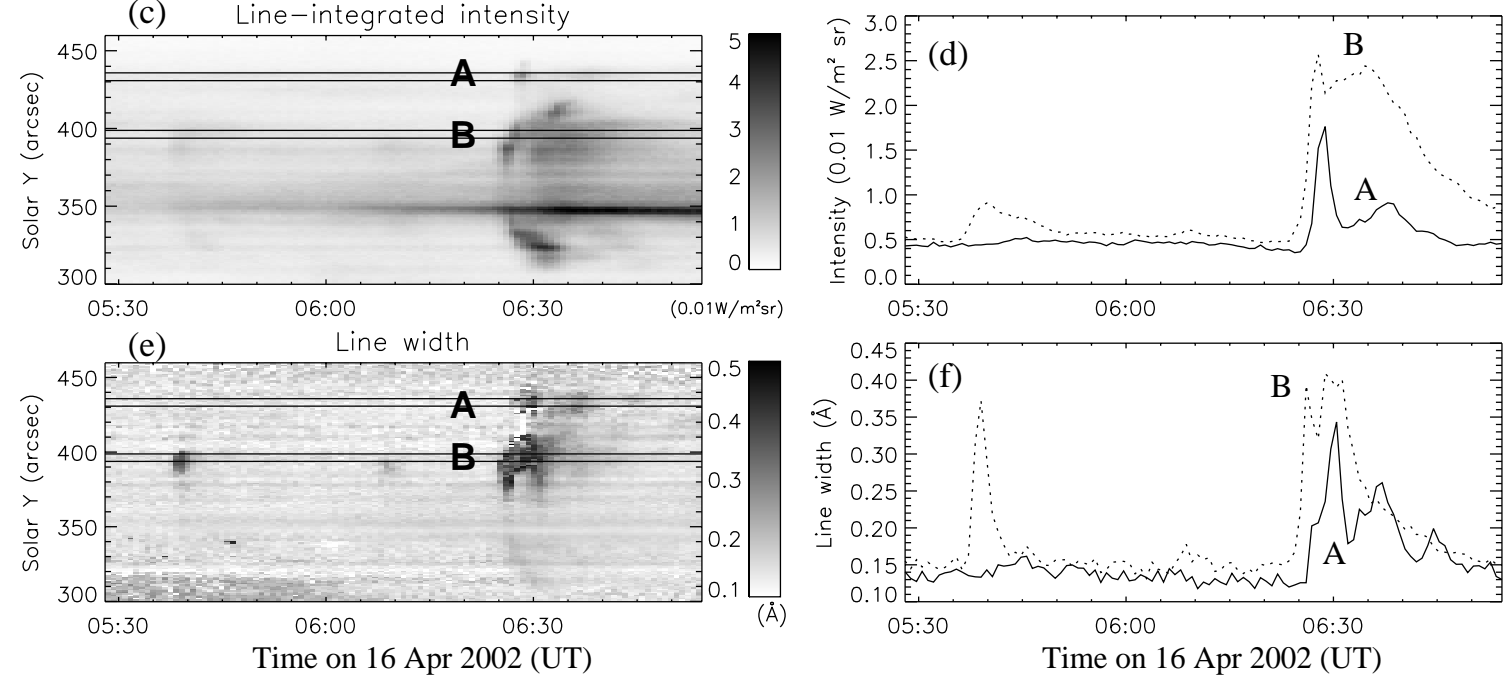

Fig. B.4. Doppler oscillation events (Nos. 19A, 19B) in the Fe XIX line on 16 April 2002. 
T. J. Wang et al.: Hot coronal loop oscillations observed by SUMER, Online Material $p 6$
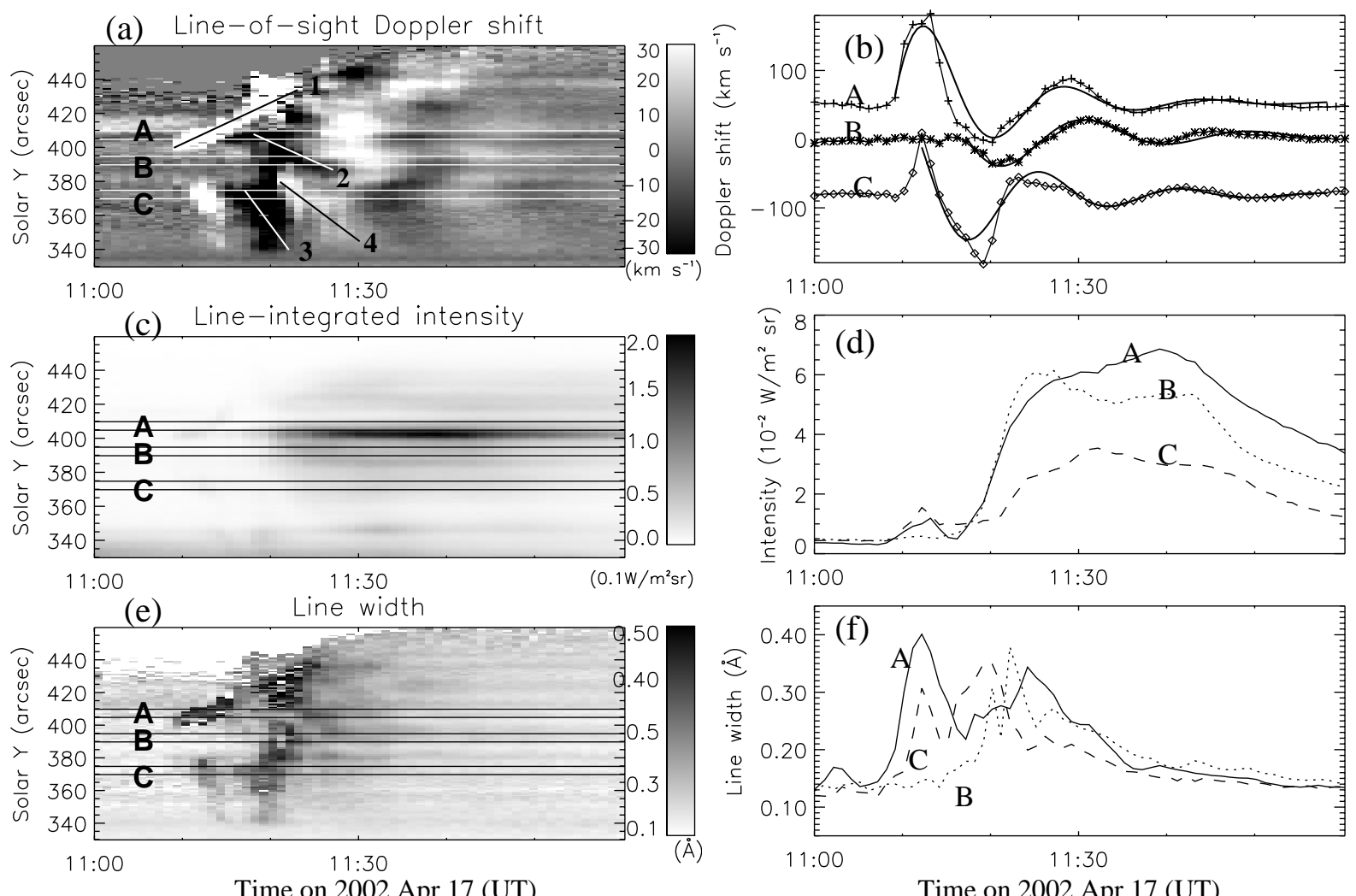

Fig. B.5. Doppler oscillation events (Nos. 20A, 20B, 20C) in the Fe XIX line on 17 April 2002. In b) the curves A and C are plotted with shifts of 50 and $-80 \mathrm{~km} \mathrm{~s}^{-1}$ along the $y$-axis, respectively.
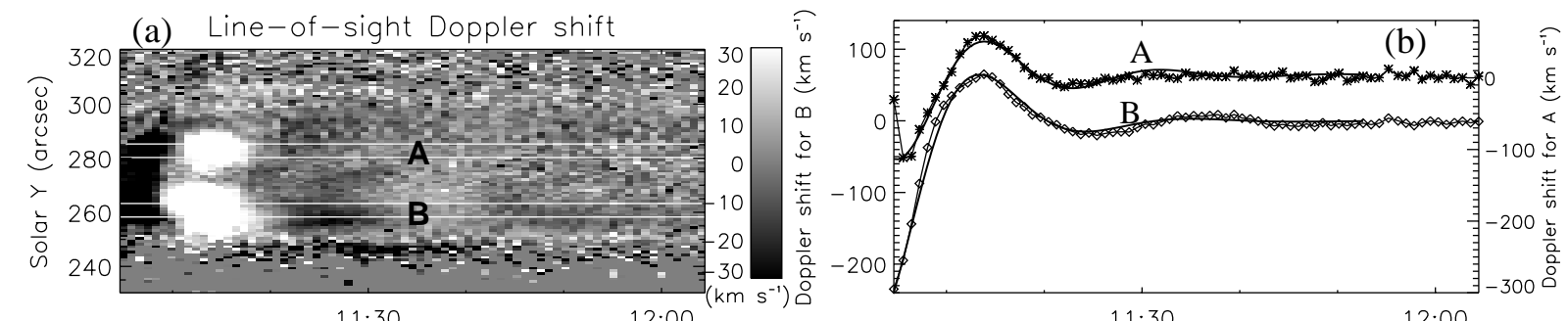

(c) Line-integrated intensity
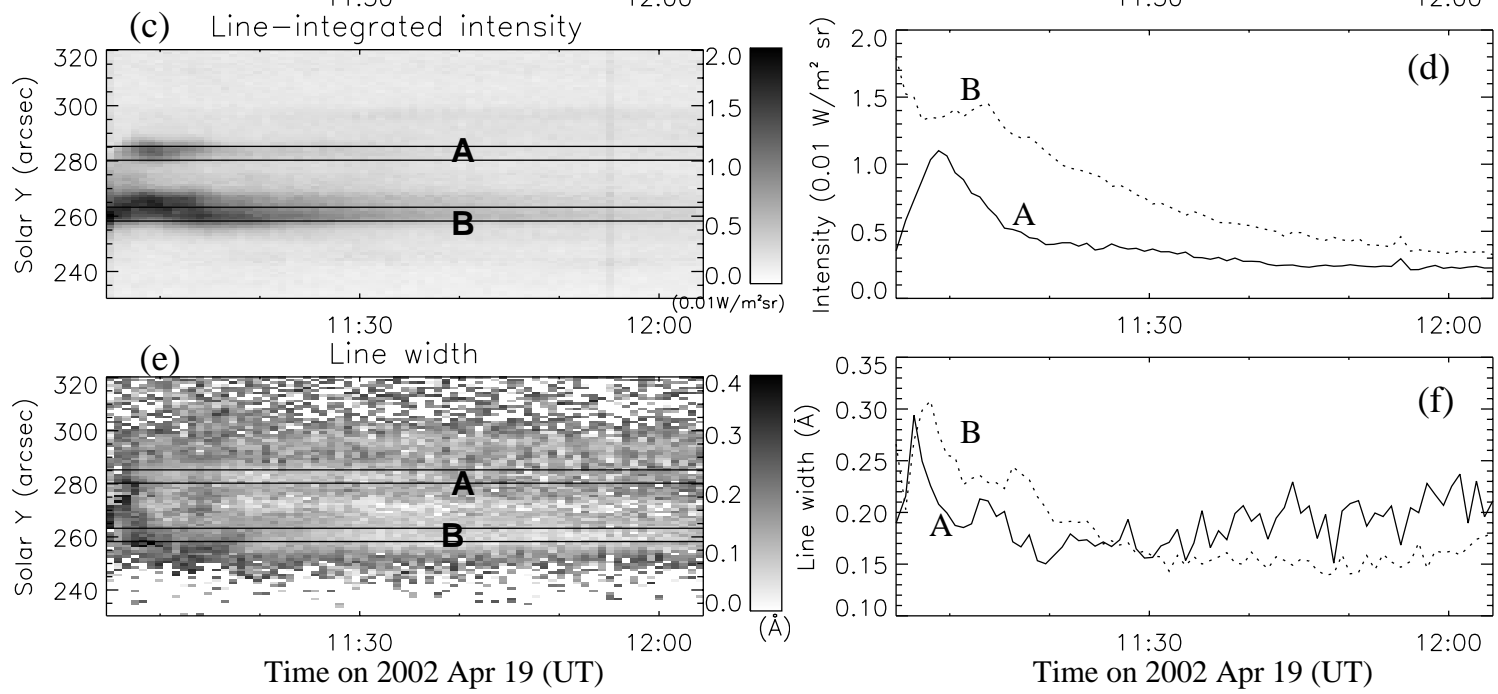

Fig. B.6. Doppler oscillation events (Nos. 21A, 21B) in the Fe XIX line on 19 April 2002. 
T. J. Wang et al.: Hot coronal loop oscillations observed by SUMER, Online Material $p 7$
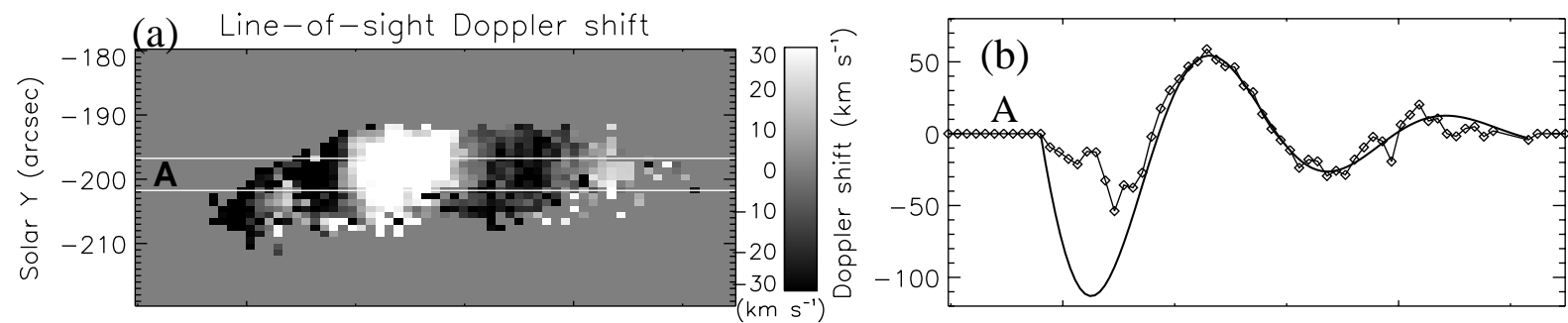

(c) Line-integrated intensity
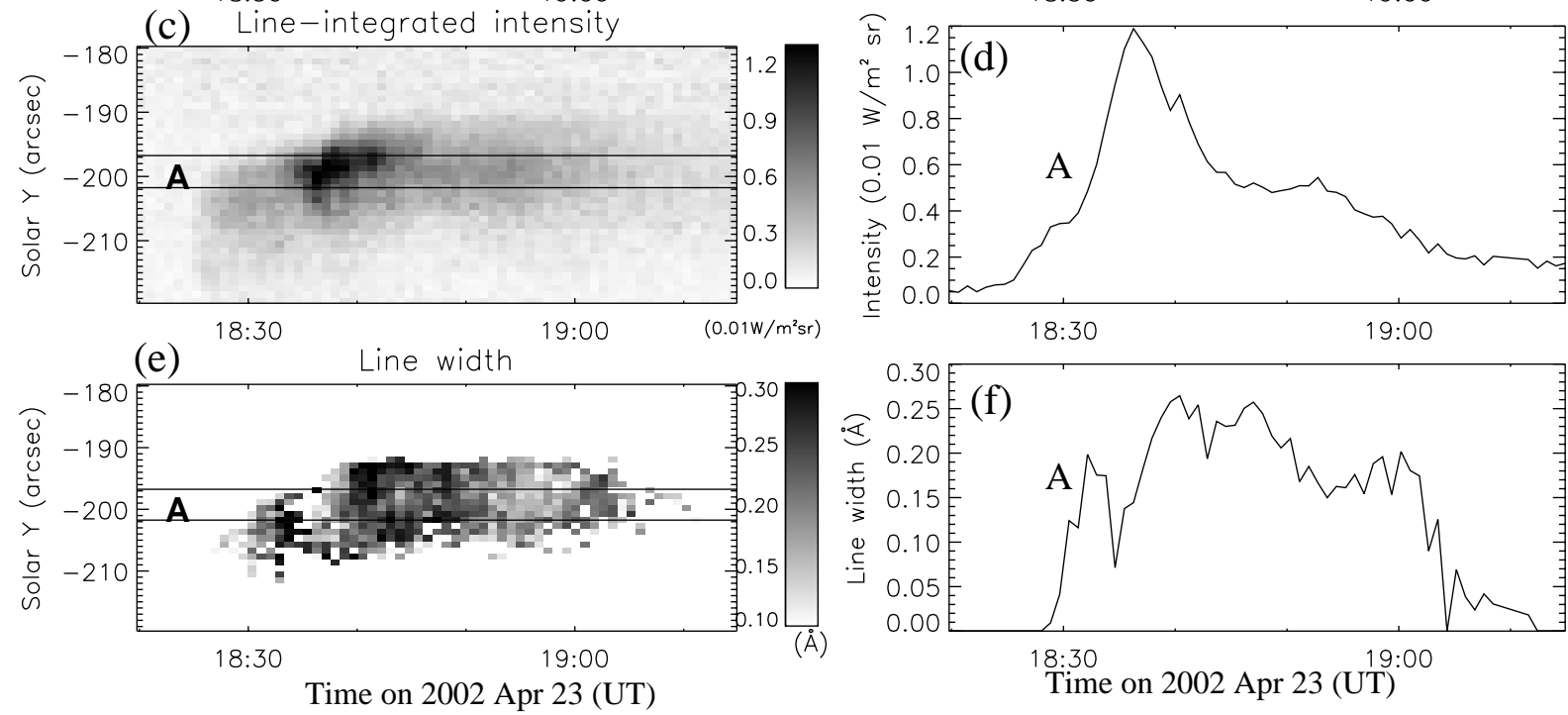

Fig. B.7. Doppler oscillation events (No. 22A) in the Fe XXI line on 23 April 2002.

(a) Line-of-sight Doppler shift

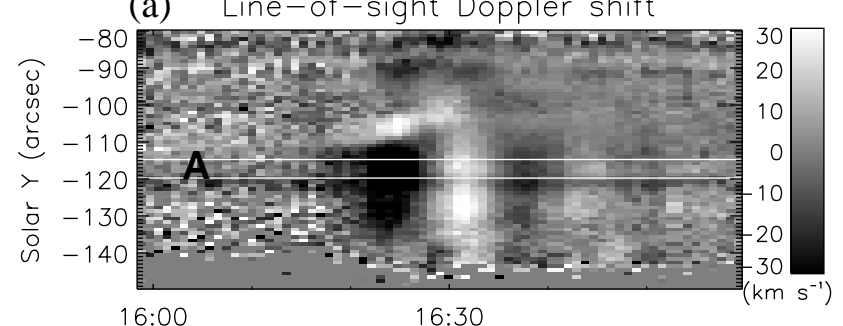

(c) Line-integrated intensity
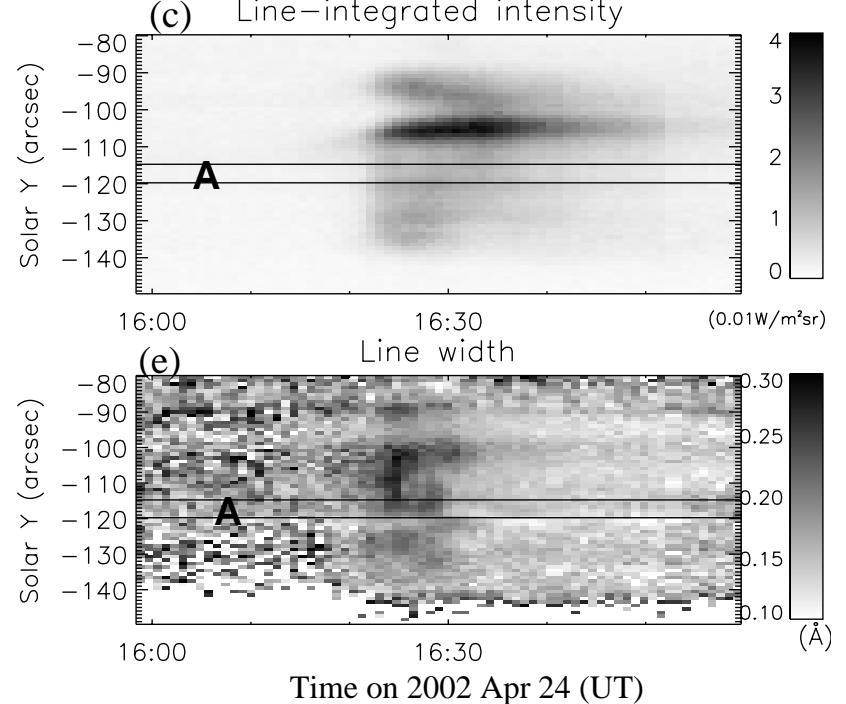
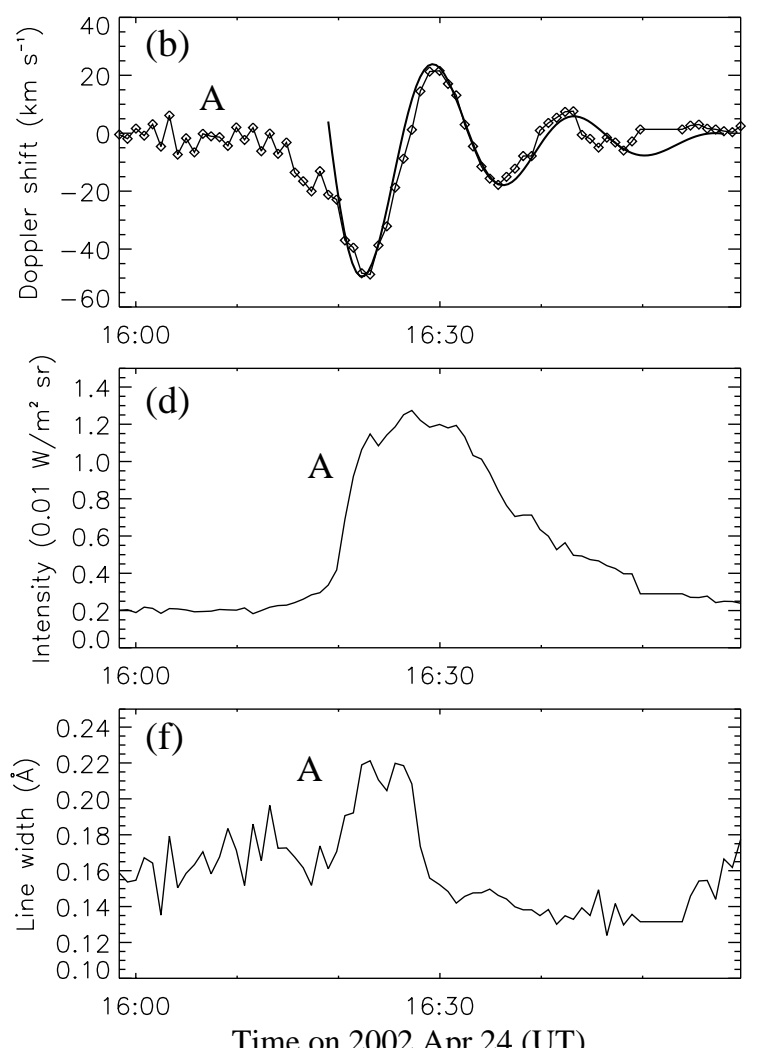

Fig. B.8. Doppler oscillation events (No. 23A) in the Fe XIX line on 24 April 2002. 
T. J. Wang et al.: Hot coronal loop oscillations observed by SUMER, Online Material $p 8$
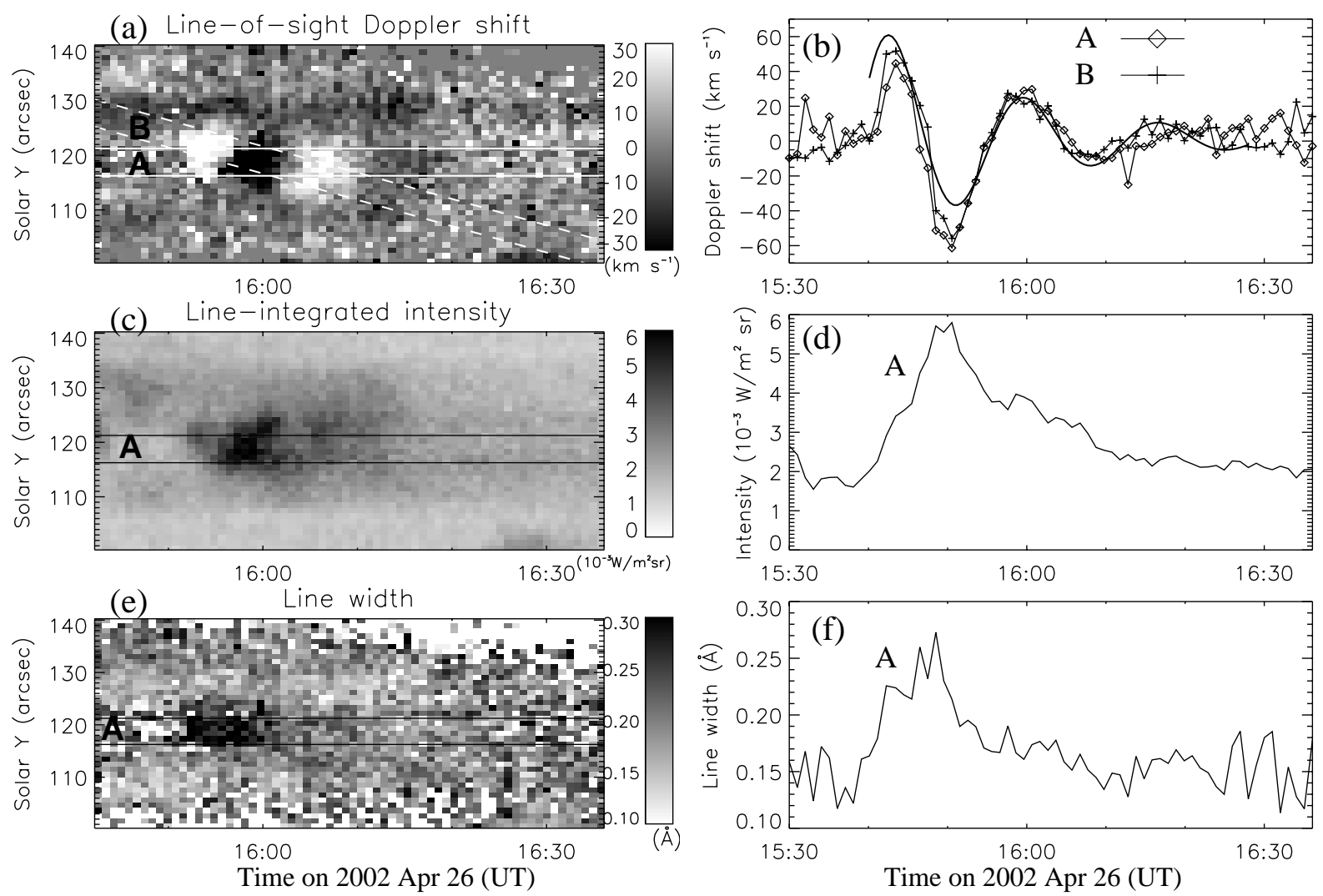

Fig. B.9. Doppler oscillation events (No. 24A) in the Fe XIX line on 26 April 2002. In a) the dashed lines show an alternative cut B for the oscillations. In $\mathbf{b}$ ) the best fit curve of the sine-function is obtained from the time profile of cut A.

(a) Line-of-sight Doppler shift
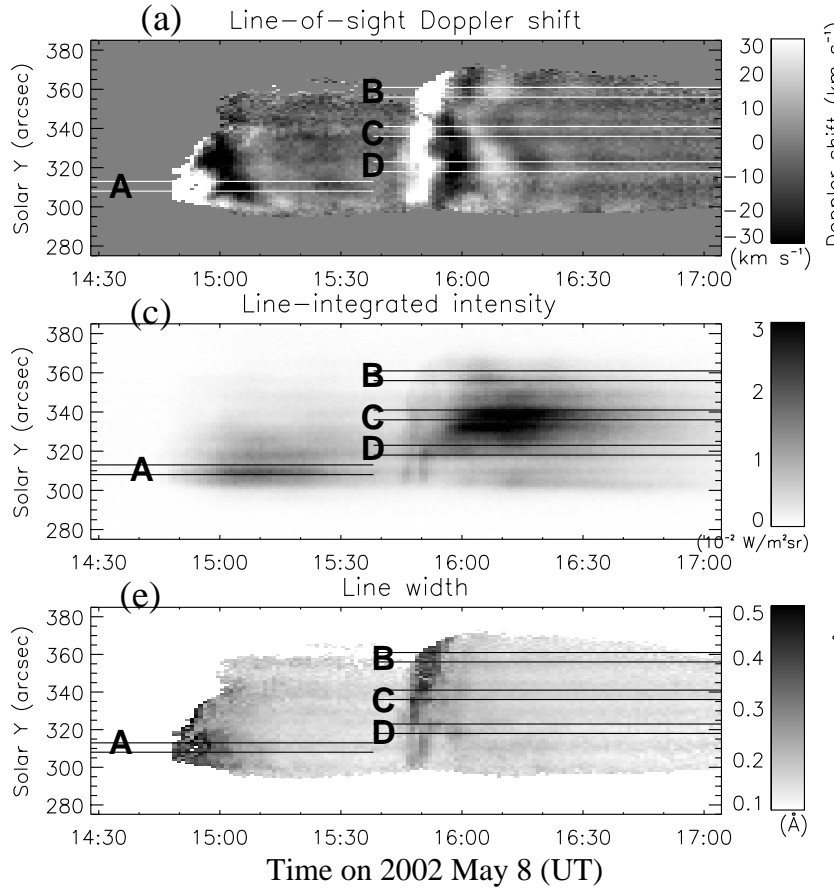
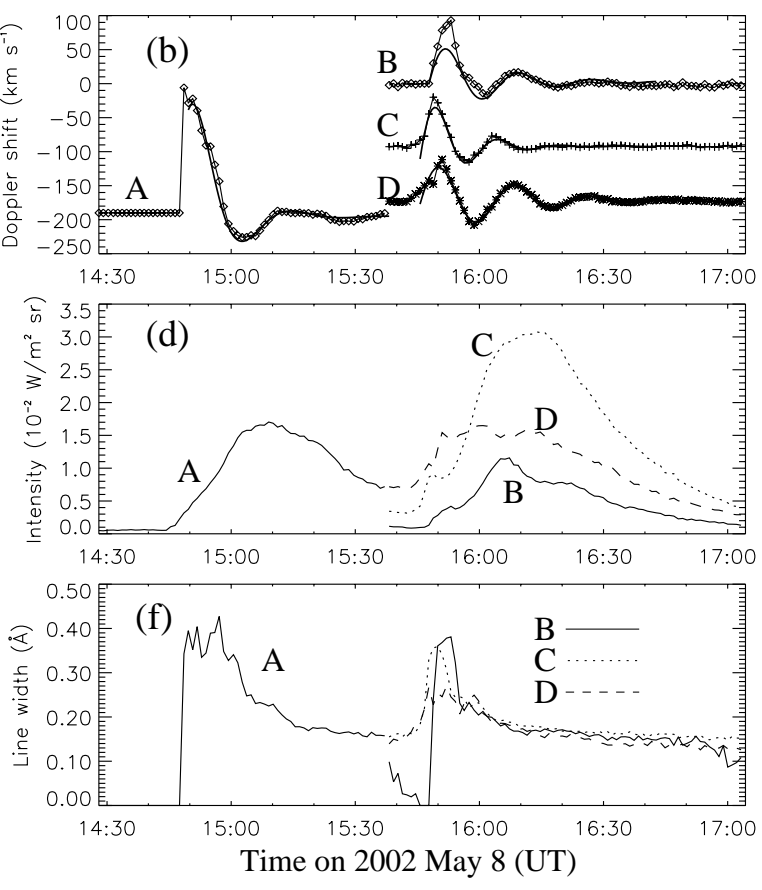

Fig. B.10. Doppler oscillation events (No. 25A, 26B, 26C, 26D) in the Fe XIX line on 8 May 2002. In b) the curves A, C and D are plotted with shifts of $-190,-90,-170 \mathrm{~km} \mathrm{~s}^{-1}$ along the $y$-axis, respectively. 
T. J. Wang et al.: Hot coronal loop oscillations observed by SUMER, Online Material $p 9$

(a) Line-of-sight Doppler shift

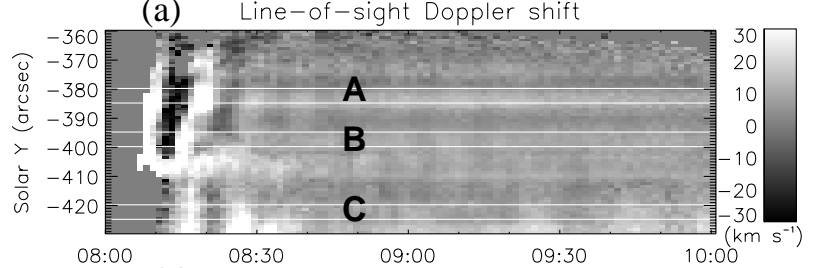

(c) Line-integrated intensity

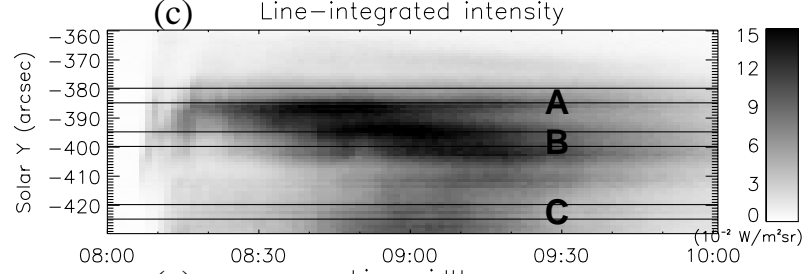

(e)

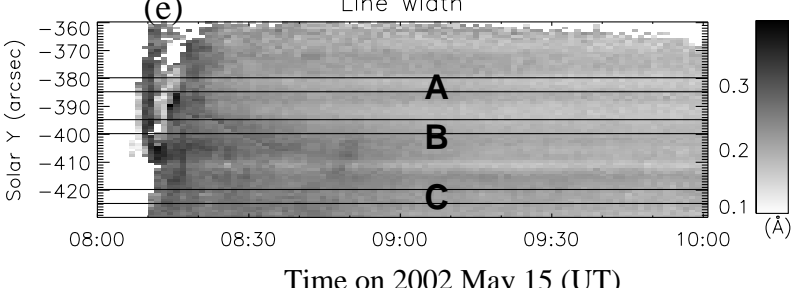

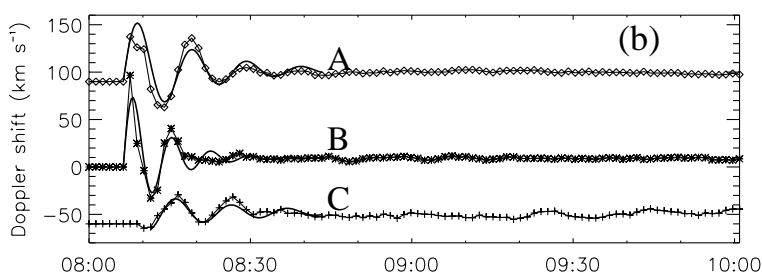
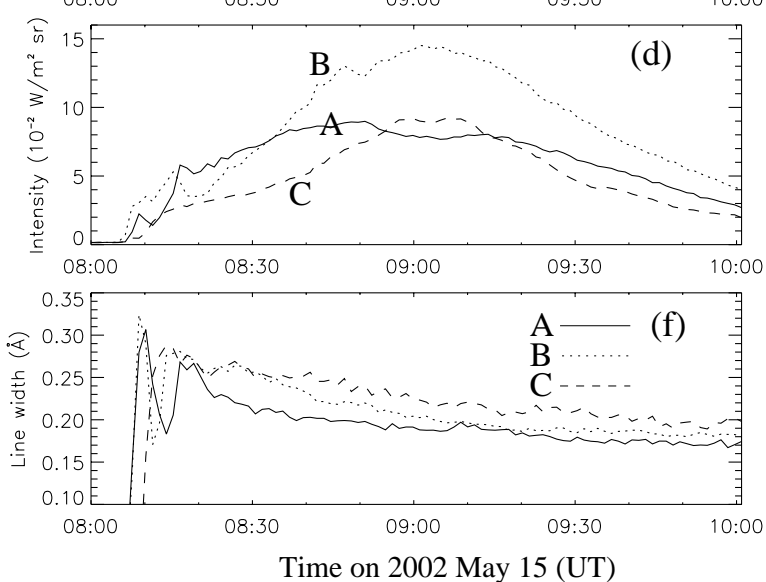

Fig. B.11. Doppler oscillation events (Nos. 27A, 27B, 27C) in the Fe XXI line on 15 May 2002. In b) the curves A and C are plotted with shifts of 90 and $-60 \mathrm{~km} \mathrm{~s}^{-1}$ along the $y$-axis, respectively. 